\title{
Scale dependence of cirrus horizontal heterogeneity effects on TOA measurements - Part I: MODIS brightness temperatures in the thermal infrared
}

\author{
Thomas Fauchez ${ }^{1,2}$, Steven Platnick ${ }^{2}$, Kerry Meyer ${ }^{3,2}$, Céline Cornet $^{4}$, Frédéric Szczap ${ }^{5}$, and Tamás Várnai ${ }^{6,2}$ \\ ${ }^{1}$ Universities Space Research Association (USRA), Columbia, MD, USA \\ ${ }^{2}$ NASA Goddard Space Flight Center, Greenbelt, MD, USA \\ ${ }^{3}$ Goddard Earth Sciences Technology and Research, Universities Space Research Association, Columbia, MD, USA \\ ${ }^{4}$ Laboratoire d'Optique Atmosphèrique, UMR 8518, Université Lille 1, Villeneuve d'Ascq, France \\ ${ }^{5}$ Laboratoire de Météorologie Physique, UMR 6016, Université Blaise Pascal, Clermont Ferrand, France \\ ${ }^{6}$ University of Maryland Baltimore County: Joint Center for Earth Systems Technology and the Department of Physics, \\ Baltimore, MD, USA \\ Correspondence to: Thomas Fauchez (thomas.j.fauchez@nasa.gov)
}

Received: 20 January 2017 - Discussion started: 7 February 2017

Revised: 16 May 2017 - Accepted: 24 May 2017 - Published: 13 July 2017

\begin{abstract}
This paper presents a study on the impact of cirrus cloud heterogeneities on MODIS simulated thermal infrared (TIR) brightness temperatures (BTs) at the top of the atmosphere (TOA) as a function of spatial resolution from $50 \mathrm{~m}$ to $10 \mathrm{~km}$. A realistic 3-D cirrus field is generated by the 3DCLOUD model (average optical thickness of 1.4, cloudtop and base altitudes at 10 and $12 \mathrm{~km}$, respectively, consisting of aggregate column crystals of $D_{\text {eff }}=20 \mu \mathrm{m}$ ), and 3-D thermal infrared radiative transfer (RT) is simulated with the 3DMCPOL code. According to previous studies, differences between 3-D BT computed from a heterogenous pixel and 1-D RT computed from a homogeneous pixel are considered dependent at nadir on two effects: (i) the optical thickness horizontal heterogeneity leading to the plane-parallel homogeneous bias (PPHB) and the (ii) horizontal radiative transport (HRT) leading to the independent pixel approximation error (IPAE). A single but realistic cirrus case is simulated and, as expected, the PPHB mainly impacts the low-spatialresolution results (above $\sim 250 \mathrm{~m}$ ) with averaged values of up to 5-7 K, while the IPAE mainly impacts the high-spatialresolution results (below $\sim 250 \mathrm{~m}$ ) with average values of up to $1-2 \mathrm{~K}$. A sensitivity study has been performed in order to extend these results to various cirrus optical thicknesses and heterogeneities by sampling the cirrus in several ranges of parameters. For four optical thickness classes and four optical heterogeneity classes, we have found that, for nadir ob-
\end{abstract}

servations, the spatial resolution at which the combination of PPHB and HRT effects is the smallest, falls between 100 and $250 \mathrm{~m}$. These spatial resolutions thus appear to be the best choice to retrieve cirrus optical properties with the smallest cloud heterogeneity-related total bias in the thermal infrared. For off-nadir observations, the average total effect is increased and the minimum is shifted to coarser spatial resolutions.

\section{Introduction}

In the context of global climate change, the representation and role of clouds are still uncertain. Cirrus clouds cover between 15 and $40 \%$ of the Earth's surface (Sassen et al., 2008) and play an important role in Earth's climate and radiative budget (Liou, 1986). The temperature difference between the cirrus cloud top and the Earth's surface leads to a warming of the atmosphere by cirrus clouds, capturing a part of the infrared radiation emitted by the atmosphere and surface. Also, cirrus clouds reflect part of the incident solar radiation into space, but this albedo effect is generally negligible for high thin clouds. Thus, on average, cirrus clouds lead to a positive radiative effect (e.g., a greenhouse effect), except for cirrus with large optical thicknesses (greater than 10; Choi and Ho, 2006) or at low altitudes (below $8 \mathrm{~km}$ in 
the tropics; Corti and Peter, 2009). The radiative impact and evolution of cirrus clouds depends on numerous factors such as cloud altitude, cloud optical and geometrical thickness, crystal shape, and effective size. Consequently, we need to improve our knowledge by improving the retrieval of cirrus cloud optical properties.

Global satellite observations are well suited to follow and better understand cloud evolution and characteristics. Therefore, many satellites are dedicated to their observations from microwave (wavelength of few millimeters) to visible ranges (wavelength up to $0.4 \mu \mathrm{m}$ ). Cirrus optical thickness (COT) and ice crystal effective diameter (CED) can be retrieved from radiometric measurements using dedicated operational algorithms. Many of these operational algorithms are developed for solar-reflectance channels, like that of the Moderate Resolution Imaging Spectroradiometer (MODIS), for the MOD06 product (Platnick et al., 2003; Yang et al., 2007) or the Clouds and the Earth's Radiant Energy System (CERES) product (Minnis et al., 2011) or the Visible Infrared Imager Radiometer Suite (VIIRS; Platnick et al., 2013). Thermal infrared (TIR) channels are currently used in the MOD06 dataset to retrieve cloud temperature-pressurealtitude (and in other datasets to retrieve ozone concentration and clear-sky temperature-moisture information). However, several studies (Cooper et al., 2007; Cooper and Garrett, 2010; Wang et al., 2011) have shown that cirrus optical properties may be retrieved with a better accuracy using a combination of TIR channels instead of visible and near-infrared (VNIR) channels (such as the Nakajima and King method, Nakajima and King, 1990), as long as the cirrus is optically thin enough (with a visible optical thickness between roughly 0.5 and 3 ) and the CED is smaller than $40 \mu \mathrm{m}$. For example the split-window technique (Inoue, 1985) applied to the Advanced Very-High-Resolution Radiometer (AVHRR; Parol et al., 1991) and the Imaging Infrared Radiometer (IIR) onboard CALIPSO (Garnier et al., 2012, 2013) is used to retrieve CED and COT from the brightness temperature difference of two different channels in the infrared atmospheric windows where gaseous absorption is small. Based on the same spectral information, an optimal estimation method (OEM; Rodgers, 2000) is used for the Atmospheric Infrared Sounder V6 (AIRS, Kahn et al., 2014, 2015) and in the research-level code of Wang et al. (2016b, a) for MODIS. Another advantage of the TIR is that measurements can also be obtained in nighttime conditions, which gives a distinct benefit compared to solarreflectance channels for developing ice cloud climatologies. However, in both VNIR and TIR optical property retrieval methods, each pixel is considered independent of its neighbors (independent pixel approximation, IPA; Cahalan et al., 1994) and fully homogeneous (plane-parallel homogeneous approximation bias, PPHB; Cahalan et al., 1994). These approximations are mostly due to time constraints on 3-D forward radiative calculations, the lack of knowledge about the sub-pixel variability and the 3-D structure of the cloud.
Many studies have been conducted in the solar spectral range to better understand the impact of cloud heterogeneities on cloud products. These studies primarily concern warm clouds such as stratocumulus (Varnai and Marshak, 2001; Zinner and Mayer, 2006; Kato and Marshak, 2009; Zinner et al., 2010; Zhang and Platnick, 2011; Zhang et al., 2012) and show that the sign and amplitude of retrieval errors depend on numerous factors, such as the spatial resolution, wavelength, geometry of observation and cloud morphology. In the TIR and for ice clouds, Hogan and Kew (2005) show that radiative transfer (RT) calculations using IPA can change the mean top of the atmosphere (TOA) radiative fluxes by $45 \mathrm{~W} \mathrm{~m}^{-2}$ in the shortwave and by $15 \mathrm{~W} \mathrm{~m}^{-2}$ in the longwave. Chen and Liou (2006) show that the broadband thermal cooling rates are increased by around $10 \%$ in 3-D RT by comparison to 1-D RT. Concerning infrared (IR) radiances or brightness temperatures (BTs), Fauchez et al. (2012, 2014) show that heterogeneity effects can significantly influence cirrus optical property retrievals at the $1 \mathrm{~km}$ scale of IIR thermal infrared observations, with potentially more than $+10 \mathrm{~K}$ on TOA BT for heterogeneous pixels, depending also on the cloud altitude. Fauchez et al. (2015) also show that these TOA BT effects result in an overestimate of the retrieved effective diameter by more than $50 \%$ for small crystals (CED under $20 \mu \mathrm{m}$ ) and underestimate the retrieved optical thickness by up to $25 \%$. These errors could significantly influence the cirrus feedbacks assumed in global atmospheric models.

The impact of cloud horizontal heterogeneity on both TOA radiation and retrieved products depends on the spatial resolution of the instrument (pixel size) and the cloud type. For example, Davis et al. (1997) show for stratocumulus clouds that the heterogeneity impact on cloud optical thickness retrieved from nadir-visible radiometric measurements is at a minimum around a pixel size of 1 or $2 \mathrm{~km}$ for a small solar zenith angle $\left(22.5^{\circ}\right)$. Higher spatial resolutions enhance the IPA error (IPAE), which increases when the photon mean path (before absorption or cloud escape) is equal to or larger than the spatial resolution. Here we refer to the word photon in the sense of a fictive light particle (FLIP; Pujol, 2015) for stochastic Monte Carlo simulations. Lower spatial resolutions have larger errors due to the homogeneous and planeparallel cloud assumption bias, which increases when the assumed-homogeneous pixel size is increased. These results are very relevant as they can allow us to estimate the average error due to cloud heterogeneity based on the spatial resolution of any spaceborne radiometer. In addition, studies such as Davis et al. (1997) that attempt to identify the spatial resolution at which the error is at a minimum can help to define the ideal spatial resolution for future instruments. However, because such studies focus only on stratocumulus clouds, which are very different from cirrus, and because they were only conducted for visible wavelength, their conclusions cannot simply be extrapolated. In this paper, we focus our attention on cirrus clouds by simulating MODIS nadir TIR measurements (at the 8.52, 11.01, 12.03 
and $13.36 \mu \mathrm{m}$ wavelengths) and compare the impact of horizontal cloud heterogeneity as a function of spatial resolution from $50 \mathrm{~m}$ to $10 \mathrm{~km}$. In Sect. 2, we present the 3DCLOUD model (Szczap et al., 2014) used to simulate a realistic cirrus case study and then we discuss the ice crystal optical property model used in MOD06, as well as the 3DMCPOL radiative transfer code (Cornet et al., 2010; Fauchez et al., 2012, 2014) used to simulate the 3-D RT inside the atmosphere in the thermal infrared. In Sect. 3 we describe the PPHB and IPAE that impact the TOA BT at nadir for our cirrus cloud. In Sect. 4 we study the impacts of cirrus heterogeneities on TOA BTs viewed from nadir as a function of the spatial resolution for the four MODIS TIR channels above. The influence of the geometry of observations is discussed in Sect. 5. Summary and conclusions are given in Sect. 6 .

\section{Simulation of a realistic cirrus cloud field case study}

\subsection{Generation of a 3-D cirrus field}

\subsubsection{Scale invariant properties}

In order to study the impact of spatial resolution on cirrus heterogeneity effects, it is important to simulate as accurately as possible the cloud inhomogeneity at the observational scale. Microphysical quantities such as liquid water content (LWC) or ice water content (IWC), optical quantities such as extinction coefficient, or radiative field quantities such as radiances, reflectances and brightness temperatures are not randomly distributed from small to large scales but often follow a power law in Fourier space (Benassi et al., 2004; Cahalan and Snider, 1989; Davis et al., 1994, 1996; Davis et al., 1997; Fauchez et al., 2014, etc.). Indeed, Kolmogorov theory (Kolmogorov, 1941) shows that in the inertial domain, where the turbulence is isotropic, and at the equilibrium with large scales, spectral energy as a function of the wave number $k$ is described by a power law spectrum $E(k)$ with an exponent $\beta \sim-5 / 3$ named spectral slope. We commonly say the $E(k)$ has scale-invariant properties as expressed by the following equation:

$E(k) \propto k^{-\beta}$.

For scales smaller than the inertial domain, viscosity phenomena smooth and homogenize the fluid movement and the spectral energy is no longer correlated with the wave number (Benassi et al., 2004). The limit is not clearly defined because of limitations due to instrument resolution. However, theoretically, it could be defined as the scale of molecular dissipation, from a few millimeters or more, depending on the turbulence intensity. The upper limit is defined as the scale where the spectrum becomes flat (uncorrelated fluctuations). This scale can vary from one cloudy field to another. From in situ LWC airborne measurements, Davis et al. $(1994,1996)$ have estimated that the horizontal LWC spectral slope has

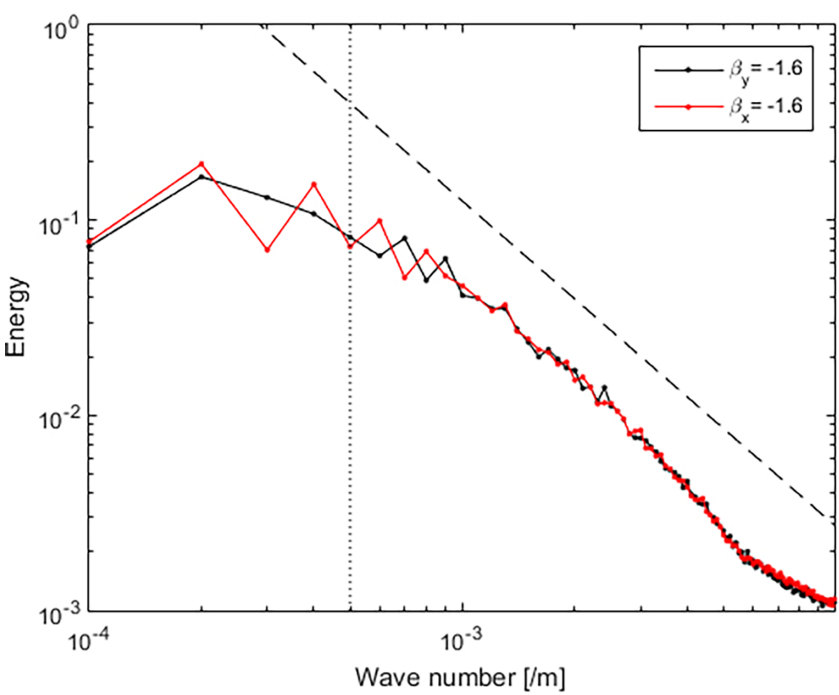

Figure 1. One-dimensionally averaged horizontal spectral slope of the cirrus following the $x$ axis (in red) and the $y$ axis (in black). The spectral slope of a $-5 / 3$ theoretical signal is drawn (dashed line). Spectral slope values, between parentheses, are estimated between $5 \times 10^{-3} \mathrm{~m}^{-1}$ wave number (vertical dotted line) and the Nyquist wave number.

a constant exponent of about $-5 / 3$ between a few meters and a few tens of kilometers for three different stratocumulus clouds. Wood and Taylor (2001) reached roughly the same conclusions for stratocumulus liquid water paths (LWPs). The situation is more complex for radiative quantities where 3-D effects (radiative smoothing and roughening) can modify the spectral slope (Oreopoulos and Cahalan, 2005). For example Cahalan and Snider (1989) have shown that in satellite measurements (particularly from the Thematic Mapper (TM) radiometer on LANDSAT), the spectral energy $E(k)$ of radiances at the TOA follow a spectral law with a $-5 / 3$ exponent from the scale of $500 \mathrm{~m}$ to about $500 \mathrm{~km}$; for scales less than $500 \mathrm{~m}$, the spectral slope decreases to values close to -3 (Davis et al., 1997).

Concerning cirrus clouds, Hogan and Kew (2005) showed using radar reflectivity that the IWC spectral slope exponent $\beta$ is equal to about $-5 / 3$ at the top of the cirrus from the scale of $1 \mathrm{~m}$ to $100 \mathrm{~km}$. However, they have also shown that the spectral slope can decrease to -3 deeper in the cirrus if its geometrical thickness is very large ( $4 \mathrm{~km}$ in their case) and the cirrus is old enough (strong sedimentation process). Wang and Sassen (2008) have also shown, for one specific cirrus case, that the spectral slope is close to $-5 / 3$ for small scales $(500 \mathrm{~m}-5 \mathrm{~km})$ but show a -3 spectral slope for larger scales $(5-100 \mathrm{~km})$. This value is explained by the authors as the consequence of different dynamic processes such as vertical wind shear, thermal stratification, and sedimentation processes and by the potentially uncommon cirrus structure. Using data from the CIRCLE-II airborne campaign, Fauchez et al. (2014) show that the horizontal spectral distribution 
(a)

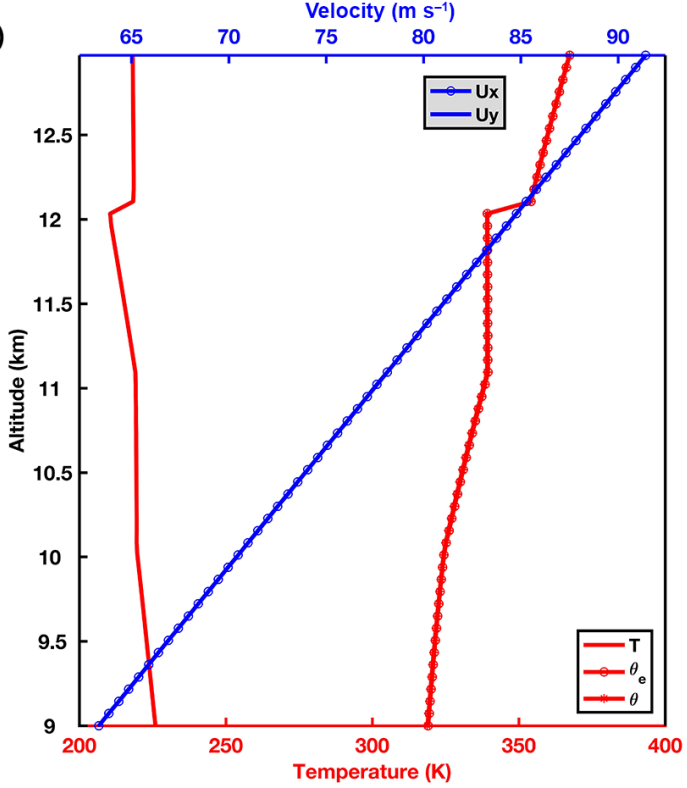

(b)

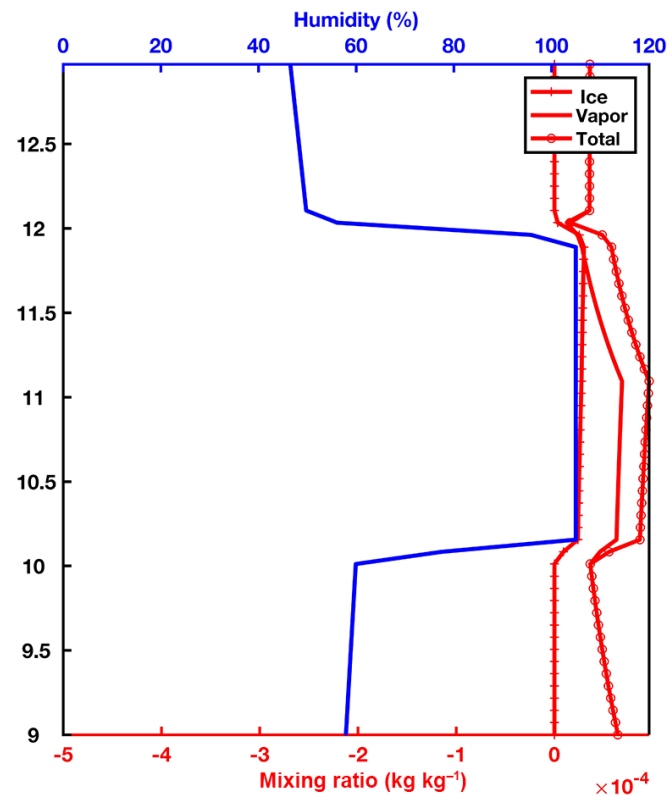

Figure 2. Meteorological profiles used to generate a realistic 3-D cirrus cloud field. (a) Wind velocity $U_{x}$ and $U_{y}$ (in blue) on the $x$ and $y$ axes, respectively; temperature $T$; potential temperature $\theta$ and equivalent potential temperature $\theta_{\mathrm{e}}$ (in red) as functions of the altitude. Panel (b) shows relative humidity (in blue), ice, vapor and total mixing ratios (in red) as functions of the altitude. Note that $U_{x}$ and $U_{y}$ are over-imposed because of wind blow at $45^{\circ}$ with respect to the $x$ and $y$ axes and $\theta$ and $\theta_{\mathrm{e}}$ are also over-imposed.

of IWC and optical thickness follow a power law with $\beta \sim$ $-5 / 3$ on the whole domain size $(20 \mathrm{~km})$. They also found the same power law at every cirrus altitude level in the $532 \mathrm{~nm}$ backscattering coefficient measured by the Cloud-Aerosol Lidar with Orthogonal Polarization (CALIOP; Winker et al., 2009). To summarize, except for particularly strong dynamical (mesoscale) processes such as those shown in Wang and Sassen (2008), the spectral slope of the horizontal distribution of IWC is typically $-5 / 3$. In this study, the size of our domain is small $(10 \times 10 \mathrm{~km})$ and mesoscale processes can thus be neglected. We therefore assume, for our simulations, that the horizontal distribution of IWC follows a power law with a $-5 / 3$ exponent at every cloud level from the smallest cloud generator scale $(50 \mathrm{~m})$ to the domain scale $(10 \mathrm{~km})$ as shown in Fig. 1.

\subsubsection{The cloud generator 3DCLOUD}

To generate 3-D cloud structures, 3DCLOUD (Szczap et al., 2014) first assimilates meteorological profiles (humidity, pressure, temperature and wind velocity) and then solves simplified basic atmospheric equations. Finally, a Fourier filtering method is used to constrain the scale-invariant properties (by imposing the horizontal 2-D distribution of IWC to follow a power law with $-5 / 3$ exponent at every cloud level) and to set the mean value and the heterogeneity parameter of these 3-D cloud structures. The heterogeneity parameter of optical thickness has been defined by Szczap et al. (2000) as $\rho_{\tau}=\sigma_{\tau} / \bar{\tau}$ with $\sigma_{\tau}$ the standard deviation of the optical thick- ness estimated for a particular pixel spatial resolution and $\bar{\tau}$ the averaged value of the optical thickness over the domain. The heterogeneity parameter is estimated without taking into account the holes in the cloud, which are already related to the fractional cover parameter (here set to 1). Fu et al. (2000), Smith and DelGenio (2001), Buschmann et al. (2002), Carlin et al. (2002), and Hogan and Illingworth (2003) have shown, using in situ or radiometric measurements, that the heterogeneity parameter $\rho_{\tau}$ is typically between 0.1 and 1.5 .

In Fig. 2 we can see the vertical profiles of the wind speed, temperature, relative humidity and ice mixing ratio assimilated by 3DCLOUD. These profiles are based on a midlatitude summer meteorological profile modified to generate cirrus clouds (see for example Szczap et al., 2014).

Figure 3 shows the optical thickness over the domain (panel a) and the 2-D IWC along the diagonal (red line in panel a) (panel b) generated using the meteorological profiles in Fig. 2 and by adjusting the optical thickness mean value while holding constant the $5 / 3$ spectral slope of the IWC power spectrum. For the cirrus used in this study, the mean optical thickness is $\tau=1.4$ at $12.03 \mu \mathrm{m}$, and the heterogeneity parameter of the optical thickness is $\rho_{\tau}=1.0$. These values are consistent with those observed for cirrus clouds as shown in Table 1, which summarizes key cirrus properties listed in the literature (Dowling and Radke, 1990; Sassen and Cho, 1992; McFarquhar and Heymsfield, 1997; Sassen et al., 2007, 2008; Szczap et al., 2014; Fu et al., 2000; Smith and DelGenio, 2001; Buschmann et al., 2002; Carlin et al., 2002; Lynch et al., 2002; Hogan and Illingworth, 2003) with the 

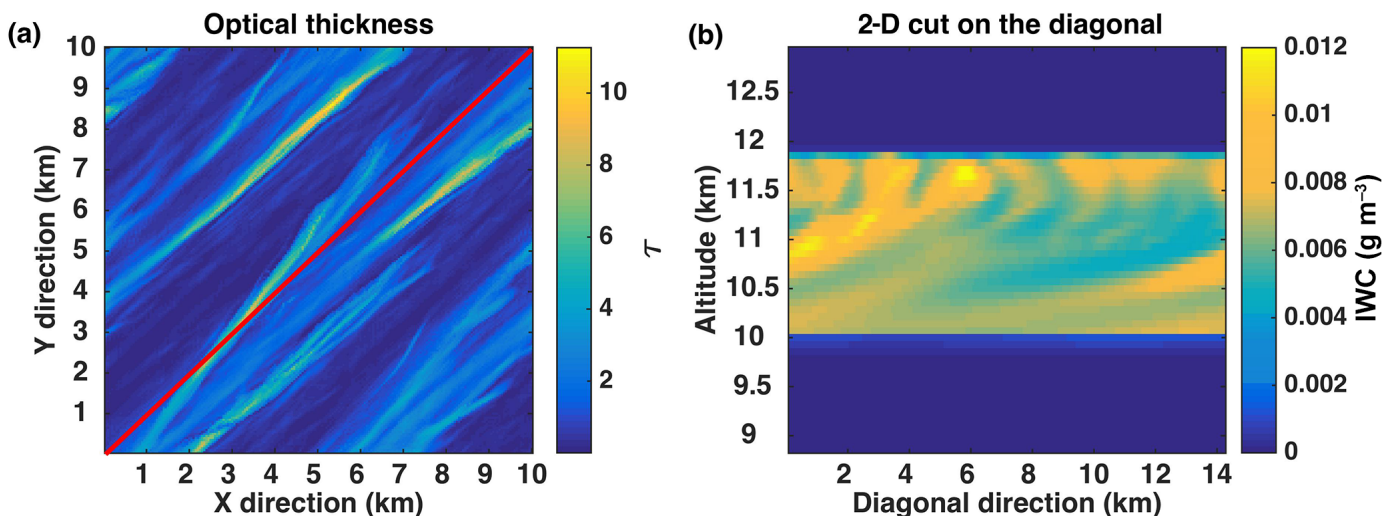

Figure 3. Panel (a) shows a $10 \times 10 \mathrm{~km}$ optical thickness ( $\tau$ at $12.03 \mu \mathrm{m}$ ) field and panel (b) shows a vertical cross section of ice water content (IWC) along the diagonal red line in (a). The mean optical thickness is 1.4 at $12.03 \mu \mathrm{m}$ and the heterogeneity parameter of the optical thickness is $\rho_{\tau}=1.0$.

Table 1. Summary of key cirrus properties from the literature based on Dowling and Radke (1990), Sassen and Cho (1992), McFarquhar and Heymsfield (1997), Sassen et al. (2007, 2008), Szczap et al. (2014), Fu et al. (2000), Smith and DelGenio (2001), Buschmann et al. (2002), Carlin et al. (2002), Lynch et al. (2002), Hogan and Illingworth (2003), etc. For each property, the range of possible values, the mean value and the value of our simulation, cirrus simu., are listed. Note that the optical thicknesses are given at $12 \mu \mathrm{m}$ and that the value in parentheses corresponds to extreme optical thickness cases for cumulonimbus plumes.

\begin{tabular}{llll}
\hline Properties & Range & Average & Cirrus simu \\
\hline Geometrical thickness $(\mathrm{km})$ & $0.1-8$ & 2 & 2 \\
Cloud-top altitude $(\mathrm{km})$ & $4-20$ & 9 & 12 \\
IWC $\left(\mathrm{g} \mathrm{m}^{-3}\right)$ & $10^{-4}-1.2$ & $2.5 \times 10^{-2}$ & $4.3 \times 10^{-3}$ \\
Crystal effective diameter $(\mu \mathrm{m})$ & $1-20$ & 40 & 20 \\
Crystal shape & Variable & Variable & Aggregate column \\
Optical thickness & $0.001-3(30)$ & 0.5 & 1.4 \\
Heterogeneity parameter of the optical thickness & $0.1-1.5$ & 0.7 & 1.0 \\
\hline
\end{tabular}

range of possible values, the mean value and the value of the simulated cirrus for each parameter. To be as realistic as possible we have chosen the properties of our simulated cirrus to be close to the average values observed in different studies (references in Table 1) and set the CED to $20 \mu \mathrm{m}$ as the sensitivity of retrievals in the thermal infrared is often limited to CED below $40 \mu \mathrm{m}$. The chosen cirrus geometry, which corresponds to an uncinus structure, is also the most common form. Two nuances should be mentioned here: (i) as seen in Table 1, most of the cirrus parameters cover a wide range of values, which means that our simulated case, while realistic in the average sense, does not represent more extreme situations. (ii) This paper is focused only on horizontal heterogeneities: we assume that the vertical variability in optical properties is negligible compared to the horizontal variability (see Fauchez et al., 2014, 2015).

\subsection{Ice crystal optical properties}

In this study, we use the same cirrus optical property coefficients as in the MOD06 product (Holz et al., 2015; Platnick et al., 2016), namely the severely roughened aggregate of solid columns of Yang et al. (2013). The selection of this particle type instead of another habit (or mixture of habits) is based on the study of Holz et al. (2015), who found that this habit provided better consistency between the IR splitwindow technique and visible and near-/shortwave-/midwave-infrared- (VNIR/SWIR/MWIR) retrieval techniques. We assume a constant crystal effective diameter of $20 \mu \mathrm{m}$ throughout the cirrus cloud. Note that TIR retrieval techniques are often limited to effective diameters between 5 and $50 \mu \mathrm{m}$. The choice of a crystal effective diameter of $20 \mu \mathrm{m}$ thus falls almost in the middle of this range. The optical properties of this ice particle as a function at each MODIS channel are shown in Table 2.

\subsection{Radiative transfer}

Radiative transfer computations are performed with the 3-D Monte Carlo code, 3DMCPOL (Cornet et al., 2010; Fauchez et al., 2012, 2014). In 3DMCPOL, the atmosphere is divided into 3-D volumes named voxels, with constant horizontal sizes $(\mathrm{d} x, \mathrm{~d} y)$ and a variable vertical size $(\mathrm{d} z)$ that depends on the atmospheric and cloud vertical stratification. Inside the 
Table 2. Bulk scattering properties (extinction coefficient $\sigma_{\mathrm{e}}$, absorption coefficient $\sigma_{\mathrm{a}}$, single-scattering albedo $\varpi_{0}$ and asymmetry parameter $g$ ) of the aggregate column ice crystal (Yang et al., 2013) with an effective diameter of $20 \mu \mathrm{m}$ for the four channels used in this study.

\begin{tabular}{|c|c|c|c|c|}
\hline & $\sigma_{\mathrm{e}}\left[\mathrm{km}^{-1}\right]$ & $\sigma_{\mathrm{a}}\left[\mathrm{km}^{-1}\right]$ & $\varpi_{0}$ & $g$ \\
\hline MODIS channel $29(8.52 \mu \mathrm{m})$ & 2.346646 & 0.594559 & 0.7466347 & 0.8643211 \\
\hline MODIS channel $31(11.01 \mu \mathrm{m})$ & 1.599258 & 0.922958 & 0.4228833 & 0.9313643 \\
\hline MODIS channel $32(12.03 \mu \mathrm{m})$ & 1.954191 & 1.028474 & 0.4737085 & 0.9126511 \\
\hline MODIS channel $33(13.36 \mu \mathrm{m})$ & 2.145600 & 1.062924 & 0.5046031 & 0.8995098 \\
\hline
\end{tabular}

cloud, each voxel is described by the cloud scattering properties: the extinction coefficient $\sigma_{\mathrm{e}}$, the single-scattering albedo $\varpi_{0}$, the phase function of the ice crystals and the temperature $T$.

3DMCPOL uses the local estimate method (LEM; Marchuk et al., 1980; Marshak and Davis, 2005; Mayer, 2009), which computes the contribution of emission, scattering or reflection events into the detector direction, attenuated by the medium optical thickness between the place of interaction and the detector (Fauchez et al., 2014). Atmospheric gaseous absorption is parameterized using a correlated $k$ distribution (Lacis and Oinas, 1991; Kratz, 1995) method combined with the equivalence theorem (Partain et al., 2000; Emde et al., 2011). The equivalence theorem is used in attaching a vector containing the atmospheric absorption attenuation to the photon package, with the vector dimension being equal to the number of bins in the correlated $k$ distribution. This allows for considerable savings in computational time.

In this study, we performed RT calculations for MODIS channels centered at $8.52,11.01,12.03$ and $13.63 \mu \mathrm{m}$ in the TIR range. 3DMCPOL directly computes the radiances, which are then converted into BTs, the quantity more commonly used in thermal infrared applications. Figure 4 shows the result of a 3-D BT computation at $12.03 \mu \mathrm{m}$ wavelength and $50 \mathrm{~m}$ horizontal spatial resolution for the cirrus scene. For this single wavelength and spatial resolution, 100 billion photon packages are computed in 10 days on the NASA Center for Climate Simulation Discover supercomputer (see acknowledgments) for an accuracy of $0.5 \mathrm{~K}$. As we will explain in Sect. 4, RT computations are performed for the different thermal infrared channels for 1-D and 3-D, and for different spatial resolutions. This yields a large number of cases and a significant computational burden. For this reason, and because Fauchez et al. (2014) showed that radiative heterogeneity effects are linked at the first order to the optical thickness heterogeneity regardless of how the optical thickness is distributed, we chose to simulate only one cirrus case. Nevertheless, the total number of simulated pixels including all wavelength channels and spatial resolutions is 313000 for the 1D simulations and 240000 for the 3-D simulations. Note that there are more 1-D computations because they are performed at various scales from $50 \mathrm{~m}$ to $10 \mathrm{~km}$ while 3-D computations are only performed at $50 \mathrm{~m}$.

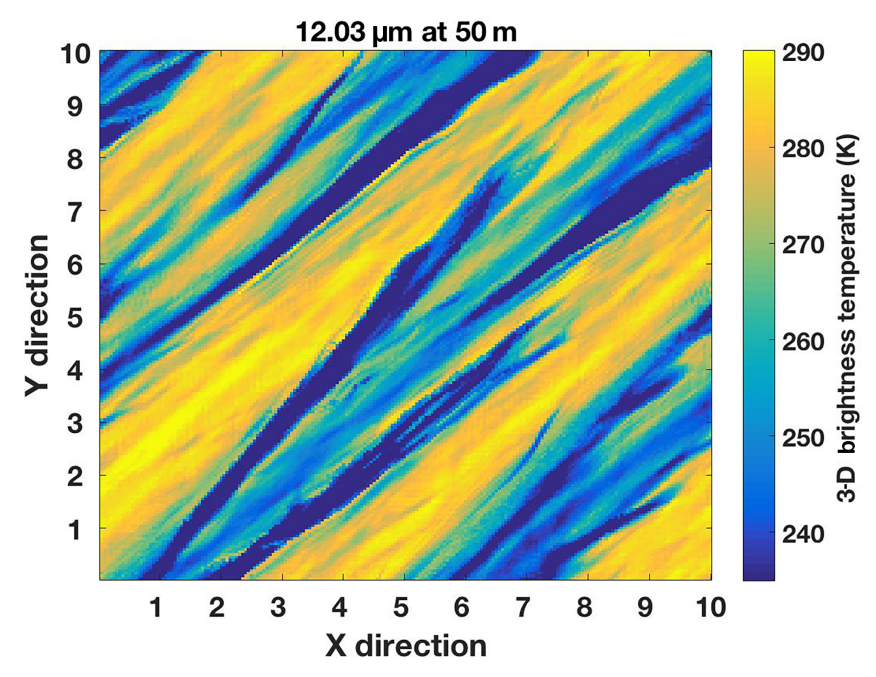

Figure 4. TOA brightness temperature field at $12.03 \mu \mathrm{m}$.

\section{Description of horizontal heterogeneity effects}

Clouds have variabilities at many different scales. However, in retrieval algorithms, for simplicity and computational reasons, the independent column approximation (ICA; Stephens et al., 1991) is commonly applied; cloud layers are assumed to be vertically and horizontally homogeneous with an infinite horizontal extent (i.e., independent of each other). From the satellite retrieval point of view, the ICA is often named IPA for independent pixel approximation (Cahalan et al., 1994). Obviously, in reality, the pixel is not homogeneous and the radiative transfer between cloudy columns occurs in 3-D. Comparisons of BT simulated with these two RT approaches (IPA and 3-D) allow us to highlight the cloud heterogeneity effects on BT.

We simulated BT with 3DMCPOL at scales ranging from $50 \mathrm{~m}$ to $10 \mathrm{~km}$. For each scale, BT values are computed using the 1-D RT assumption at the averaged COT $\left(\mathrm{BT}_{\mathrm{km}}^{1-\mathrm{D}}\right.$ with $x$ as the scale and kilometers as the distance unit) and compared with 3-D radiance simulations at the finest field spatial resolution $(50 \mathrm{~m})$, arithmetically averaged to the scale being considered and converted to BT (for simplification reasons, we will refer to this process as BT averaging). The latter are noted as $\overline{\mathrm{BT}_{50 \mathrm{~m}}^{3-\mathrm{D}}} \times \mathrm{km}$. The choice of the native spatial resolution for 3-D computations should be much smaller than 
Table 3. Average number of scattering and photon mean horizontal displacements (photon mean path) as a function of the optical thickness for channels centered at 8.52, $11.01,12.03$ and $13.36 \mu \mathrm{m}$.

\begin{tabular}{lrrrr|rrrr}
\hline Optical thickness & \multicolumn{3}{c|}{$\begin{array}{c}\text { Average number of scattering } \\
\text { wavelength }\end{array}$} & \multicolumn{4}{c}{$\begin{array}{c}\text { Photon mean path }(\mathrm{km}) \\
\text { wavelength }\end{array}$} \\
\cline { 2 - 9 } & $8.52 \mu \mathrm{m}$ & $11.01 \mu \mathrm{m}$ & $12.03 \mu \mathrm{m}$ & $13.36 \mu \mathrm{m}$ & $8.52 \mu \mathrm{m}$ & $11.01 \mu \mathrm{m}$ & $12.03 \mu \mathrm{m}$ & $13.36 \mu \mathrm{m}$ \\
\hline 1 & 1.43 & 0.87 & 1.02 & 1.11 & 3.34 & 2.93 & 2.68 & 2.59 \\
2 & 2.28 & 1.27 & 1.45 & 1.55 & 2.11 & 1.77 & 1.60 & 1.54 \\
5 & 3.17 & 1.56 & 1.71 & 1.81 & 1.00 & 0.78 & 0.69 & 0.66 \\
7 & 3.29 & 1.58 & 1.73 & 1.82 & 0.72 & 0.56 & 0.50 & 0.48 \\
10 & 3.33 & 1.59 & 1.73 & 1.82 & 0.51 & 0.40 & 0.35 & 0.33 \\
\hline
\end{tabular}

(a)

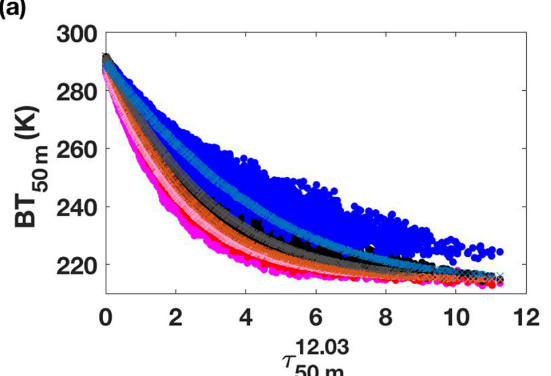

(c)

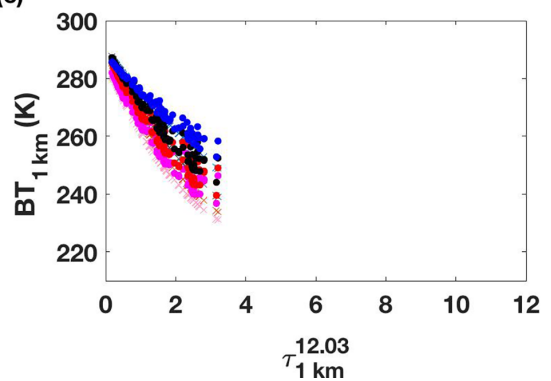

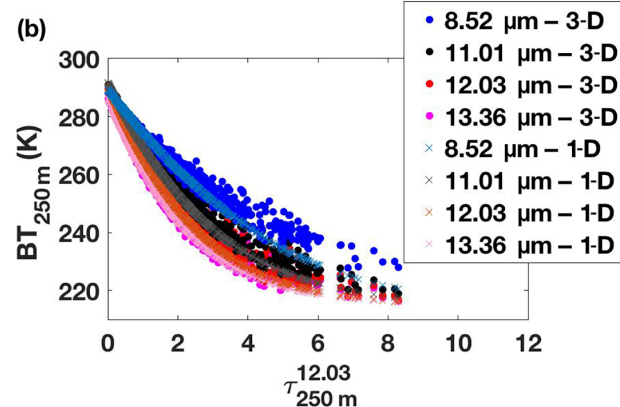

(d)

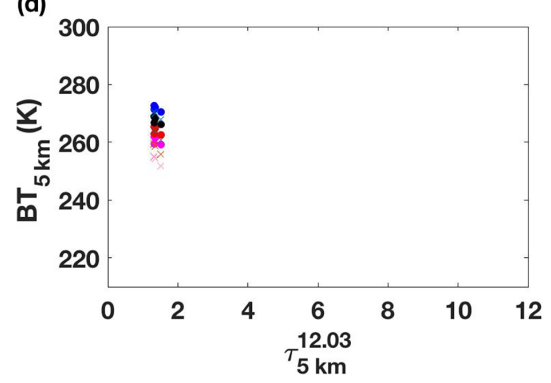

Figure 5. Brightness temperature (BT) as a function of the optical thickness at $12.03 \mu \mathrm{m}$ for MODIS channels centered at $8.52,11.01,12.03$ and $13.36 \mu \mathrm{m}$ at spatial resolutions of $50 \mathrm{~m} \mathrm{(a),} 250 \mathrm{~m} \mathrm{(b),} 1 \mathrm{~km}(\mathbf{c})$ and $5 \mathrm{~km}(\mathbf{d})$.

the photon mean path (distance traveled before absorption or cloud escape) to account for horizontal radiative transport effects. However, the finer the spatial resolution is, the more pixels can communicate. The finer spatial resolution 3DMCPOL can achieve in a reasonable computational time for $10 \mathrm{~km}$ domain is $50 \mathrm{~m}$. Table 3 summarizes the number of scattering and photon mean paths computed using Marshak and Davis (2005) (chapter 12) for various optical thicknesses and for channels centered at 8.52,11.01, 12.03 and $13.63 \mu \mathrm{m}$. Note that the number of scatterings increases with the optical thickness and is almost twice as large at $8.52 \mu \mathrm{m}$. Obviously the photon mean geometrical path decreases with optical thickness (for the same cloud geometry) and is about $3 \mathrm{~km}$ at $8.52 \mu \mathrm{m}$ for an optical thickness of 1 and only about $0.5 \mathrm{~km}$ for an optical thickness of 10 .

In Fig. 5, we plot 1-D and 3-D BTs as a function of the optical thickness at $12.03 \mu \mathrm{m}$ and at the spatial resolution of
$50 \mathrm{~m}$ (panel a), $250 \mathrm{~m}$ (panel b), $1 \mathrm{~km}$ (panel c) and $5 \mathrm{~km}$ (panel d) for the four MODIS TIR channels. For a better readability, 1-D cases are a color tone lighter than their corresponding 3-D case. First, we see that 3-D and 1-D BTs decrease as optical thickness increases because the warmer surface contributes less to the signal as the cloud becomes more opaque. Also, the relationship between the BT and the optical thickness is nonlinear and depends on the optical thickness. This is particularly clear for the highest spatial resolution $(50 \mathrm{~m})$ where no averaging of the 3-D BTs has been performed. Two effects explain the differences between 3-D and 1-D BTs: the plane-parallel and homogeneous bias and the horizontal radiative transport.

\subsection{Plane-parallel and homogeneous bias (PPHB)}

The relationship between BT and the optical thickness is nonlinear, leading to the Jensen inequality, and is usually re- 


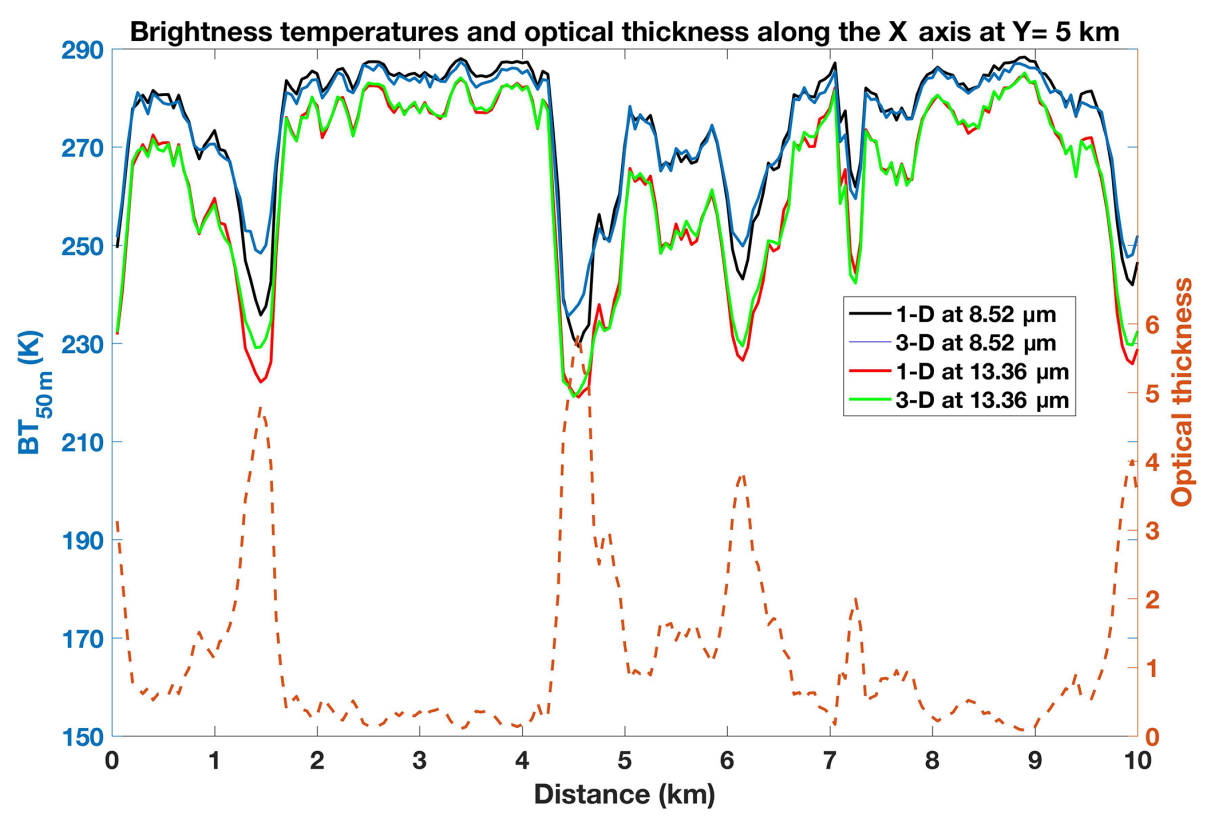

Figure 6. Optical thickness and brightness temperatures at $50 \mathrm{~m}$ spatial resolution computed in $3-\mathrm{D}$ and $1-\mathrm{D}$ at 8.52 and $13.36 \mu \mathrm{m}$ along a line of constant $y$ axis coordinate $(5 \mathrm{~km})$ in the optical thickness field of Fig. $3 \mathrm{a}$.

ferred to as the PPHB (Cahalan et al., 1994). When BTs are averaged from a high spatial resolution to a coarse spatial resolution, the average $\mathrm{BT}$ is different from the $\mathrm{BT}$ of the average optical thickness. In the thermal infrared, the averaged $\mathrm{BT}$ is larger than the BT directly computed from the average optical thickness. The PPHB is observed at all spatial resolutions $(50 \mathrm{~m}, 250 \mathrm{~m}, 1 \mathrm{~km}$ and $5 \mathrm{~km}$ in Fig. $5 \mathrm{a}, \mathrm{b}, \mathrm{c}$ and d, respectively) and for coarsening resolution. The average BT3D is larger than the corresponding BT1D as predicted by the Jensen inequality for the curvature of the relation.

For cirrus clouds observed in the thermal infrared from nadir, Fauchez et al. $(2012,2014)$ have shown that at a $1 \mathrm{~km}$ spatial resolution, the PPHB is the main heterogeneity effect and is essentially correlated (around 98\%) with three parameters:

- the standard deviation $\sigma_{\tau}$ of the optical thickness inside the observation pixel

- the brightness temperature contrast $(\triangle \mathrm{BT}(\mathrm{CS}-\mathrm{OP}))$ between the clear sky (CS) and an opaque cloudy pixel (OP)

- the effective size of ice crystals (in the range $D_{\text {eff }}=5-$ $30 \mu \mathrm{m}$ where the absorption varies significantly).

Because cirrus clouds can be very heterogeneous (Sassen and Cho, 1992; Carlin et al., 2002; Lynch et al., 2002) and their cloud-top altitude very high $(5-20 \mathrm{~km})$, the impact of the cloud horizontal heterogeneity on TOA BT can reach more than $15 \mathrm{~K}$ for heterogeneous cirrus cloud pixels of $1 \times 1 \mathrm{~km}$ at about $10 \mathrm{~km}$ altitude (Fauchez et al., 2014). It can probably be larger for tropical or equatorial cirrus for which the altitude can be higher than $15 \mathrm{~km}$ and the surface temperature higher than $310 \mathrm{~K}$. In this situation the contrast between the clear sky BT and opaque cloudy pixel BT is large, leading to a likewise large PPHB. Obviously, such a BT bias can severely impact a cloud-top property retrieval (emissivity, cloud-top height, etc.).

\subsection{Horizontal radiative transport (HRT)}

In addition to the PPHB, the IPAE can also impact TOA BT through horizontal radiative transport (HRT). This effect is small in the TIR at a scale of $1 \mathrm{~km}$ but not necessary at a $50 \mathrm{~m}$ spatial resolution, which is smaller than the photon mean path. Indeed, as seen in the different subplots of Fig. 5, 1-D calculations show a one-to-one relationship between BT and optical thickness, but the 3-D relation is highly dispersed because of HRT effects between cloudy columns. In addition, points are less scattered at the coarsest spatial resolutions (1 and $5 \mathrm{~km}$ ), which means that the HRT effect is reduced; the number of points are of course also reduced by averaging to coarser resolution.

In Fig. 6, we can see 3-D and 1-D BT, computed at $50 \mathrm{~m}$ resolution along a line parallel to the $X$ dimension in Fig. 3a at a $Y$ coordinate of $5 \mathrm{~km}$ for channels centered at 8.52 and $13.36 \mu \mathrm{m}$; also shown is the optical thickness at $12.03 \mu \mathrm{m}$. For both channels, 1-D BT is larger than 3-D BT when the optical thickness is small and vice versa when the optical thickness is large. This means that, on average, extreme values of 3-D BT are reduced by HRT smoothing. The 3-D BT field looks more homogeneous than the 1-D BT field where 
(a)

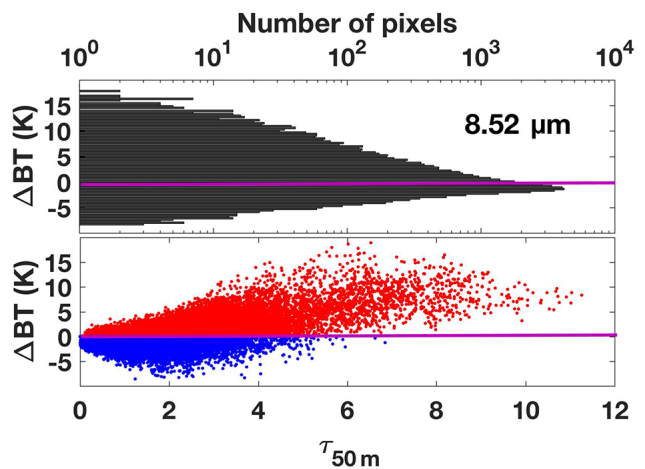

(c)

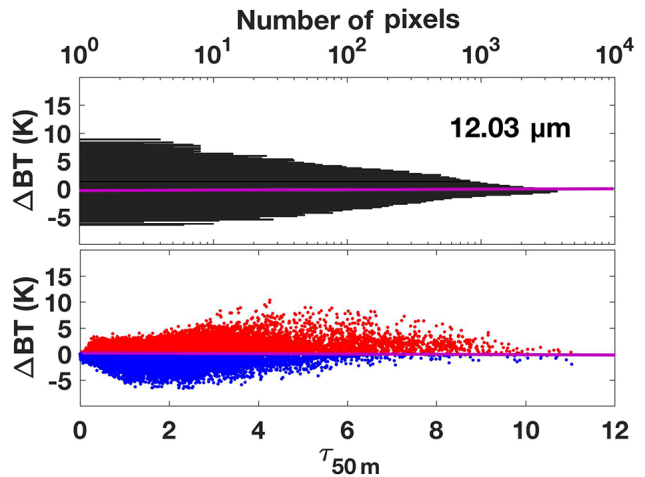

(b)

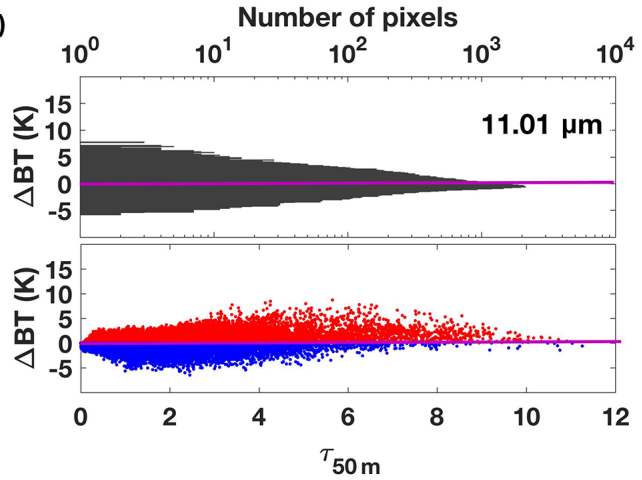

(d)

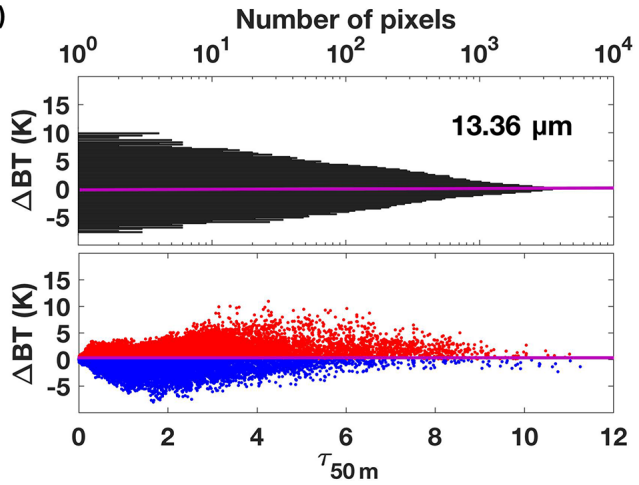

Figure 7. The contribution of the photon horizontal transport to TOA brightness temperature differences between 3-D and 1-D RT at 50 $\mathrm{m}$ $\left(\triangle \mathrm{BT}=\mathrm{BT}_{50 \mathrm{~m}}^{3-\mathrm{D}}-\mathrm{BT}_{50 \mathrm{~m}}^{1-\mathrm{D}}\right)$ seen from nadir as a function of the optical thickness at $12.03 \mathrm{~m}$ (bottom frame). The fraction of pixel for each $\triangle \mathrm{BT}$ is shown in the top frame. Positive and negative differences are in red and blue, respectively, at $8.52 \mu \mathrm{m}(\mathbf{a}), 11.01 \mu \mathrm{m}(\mathbf{b}), 12.03 \mu \mathrm{m}(\mathbf{c})$ and $13.36 \mu \mathrm{m}(\mathbf{d})$. For these four channels, the $\Delta \mathrm{BT}$ percentage of positive values are 33, 40, 41 and $53 \%$, respectively.

no smoothing occurs. Because this difference is amplified with the number of scatterings, the differences between 3$\mathrm{D}$ and 1-D for the channel at $8.52 \mu \mathrm{m}$ are stronger than at $13.36 \mu \mathrm{m}$, particularly for large optical thicknesses, a tendency that will impact cloud optical property retrievals that use a combination of these channels.

Figure 7 shows the brightness temperature differences $\Delta \mathrm{BT}=\mathrm{BT}_{50 \mathrm{~m}}^{3-\mathrm{D}}-\mathrm{BT}_{50 \mathrm{~m}}^{1-\mathrm{D}}$ and their distribution for each $50 \times 50 \mathrm{~m}$ pixel of the $10 \times 10 \mathrm{~km}$ field versus the number of pixels (top panels) and optical thickness (lower panels) for MODIS channels centered at $8.52 \mu \mathrm{m}$ (panel a), $11.01 \mu \mathrm{m}$ (panel b), $12.03 \mu \mathrm{m}$ (panel c) and $13.36 \mu \mathrm{m}$ (panel d). Positive values are shown in red, negative ones in blue. Because BT values from 3-D and 1-D RT are computed at the same spatial resolution $(50 \mathrm{~m})$, there is no horizontal heterogeneity effect (no PPHB); only the HRT effect occurs. We can see that the largest values of $\Delta \mathrm{BT}$ are at $8.52 \mu \mathrm{m}$ because of the larger single-scattering albedo leading to more scattering. For this channel, $\triangle \mathrm{BT}$ ranges from -9 to $+19 \mathrm{~K}$ (top panel in a) and is very dependent on $\tau_{50 \mathrm{~m}}^{12}$ (bottom panel in a). Indeed, (i) the largest $\tau_{50 \mathrm{~m}}^{12}$ preferentially lead to 3D BT > 1-D BT because, as seen in Fig. 6, scattered photons coming from small optical thicknesses (associated with largest BTs) drastically increase the BT of larger optical thicknesses through HRT. This effect is particularly notice- able for $\tau_{50 \mathrm{~m}}^{12}>6$ in which only positive $\Delta \mathrm{BT}$ exist. However, for very large values, absorption is so strong that the $\Delta \mathrm{BT}$ increase is mitigated. (ii) For the smallest $\tau_{50 \mathrm{~m}}^{12}$ values (below 3), negative $\Delta \mathrm{BT}$ values dominate because photons coming from thick and cold areas decrease the BT of these pixels (see Fig. 6). The minimum $\Delta \mathrm{BT}$ is around $\tau_{50 \mathrm{~m}}^{12}=2$. Below this value, the efficiency of the HRT effect is reduced by the decrease in cloud extinction. BTs are dominated by the surface emission, reducing the BT contrast between smaller and larger $\tau_{50 \mathrm{~m}}^{12}$ values, and the chance of scattering is weak, leading to a small HRT effect.

The $\triangle \mathrm{BT}$ s are smaller in channels at $11.01,12.03$ and $13.63 \mu \mathrm{m}$ and they are more symmetric with respect to $0 \mathrm{~K}$. This greater symmetry is due to less scattering and larger absorption in the cloud (see optical properties in Table 2). This is particularly clear at $11.01 \mu \mathrm{m}$ where cloud extinction is significantly smaller than in the other channels, reducing the probability of scattering and thus the amplitude of the HRT effect, even if the photons can propagate farther in the cloud. For the three channels, below $\tau_{50 \mathrm{~m}}^{12}=3$, the HRT effect from large to small $\tau_{50 \mathrm{~m}}^{12}$ pixels tends to dominate, leading on average to $(\overline{\Delta \mathrm{BT}}<0)$. However, for larger optical thicknesses, it is the HRT effect from small to large $\tau_{50 \mathrm{~m}}^{12}$ that dominates, leading on average to $(\overline{\Delta \mathrm{BT}}>0)$. Contrary 
to the channel at $8.52 \mu \mathrm{m}$, photons coming from small $\tau_{50 \mathrm{~m}}^{12}$ propagate less to very large $\tau_{50 \mathrm{~m}}^{12}$ because of the stronger absorption. In addition, the brightness temperature between large optical thicknesses is quite similar $(\sim 215 \mathrm{~K})$. Therefore, very large $\tau_{50 \mathrm{~m}}^{12}$ values are hardly impacted by the photon transport (only $\pm 2 \mathrm{~K}$ due to neighboring pixels with a similar $\tau_{50 \mathrm{~m}}^{12}$ ). As a result, the maximum of $\Delta \mathrm{BT}$ is around $\tau_{50 \mathrm{~m}}^{12}=5$. Note that for all the channels, the field-averaged error in $\overline{\triangle \mathrm{BT}}$ due to HRT is almost nil. For the channel centered at $13.36 \mu \mathrm{m}, \overline{\Delta \mathrm{BT}}$ is slightly positive $(0.15 \mathrm{~K})$, while for the other channels it is slightly negative $(\sim-0.06 \mathrm{~K})$. The reason why $\overline{\Delta \mathrm{BT}}$ is positive at $13.36 \mu \mathrm{m}$ and negative for the other channels is due to the larger absorption optical thickness at $13.36 \mu \mathrm{m}$ causing a HRT effect dominated by the effect described above (ii). Note that, according to MOD06 ice radiative models, the single-scattering albedo of large ice crystals in the other channels will converge to values close to those of the $13.36 \mu \mathrm{m}$ at $\mathrm{CED}=20 \mu \mathrm{m}$. Therefore, the HRT in the three other channels will be similar to that of the channel centered at $8.52 \mu \mathrm{m}$.

This shows that the effect of both PPHB and HRT on TOA BT strongly depends on the spatial resolution, as discussed in the next section.

\section{Horizontal heterogeneity effects as functions of the nadir-observed scale}

As discussed in Sect. 3, heterogeneity effects on the radiative fields observed from nadir at TOA depend, on the one hand, on the sub-pixel optical thickness inhomogeneity (PPHB) and on the other hand, on the IPAE (HRT effect). The optimal resolution for cloud retrievals is therefore a compromise between reducing the PPHB by improving the spatial resolution but not causing larger increases in HRT effect. The objective is thus to find the smallest spatial resolution that strikes a balance between the PPHB and the absolute error due to the HRT. This spatial resolution depends of course on the wavelength (dependence on the photon mean path), cloud type (different optical properties, optical and geometrical thicknesses, and altitude) and the geometry of observation.

The total difference, computed as the total arithmetic mean difference (AMD) between averaged 3-D and non-averaged 1-D TOA BT viewed from nadir as a function of the spatial resolution, is given by

$$
\begin{aligned}
& \operatorname{AMD}\left(\overline{\left.\Delta \mathrm{BT}_{x \mathrm{~km}}^{3-\mathrm{D}-1-\mathrm{D}}\right)=}\right. \\
& {\left[\sum_{i=1}^{N}\left({\overline{\mathrm{BT}_{50 \mathrm{~m}}^{3-\mathrm{D}}}}^{x \mathrm{~km}}-\mathrm{BT}_{x \mathrm{~km}}^{1-\mathrm{D}}\right)\right] / N,}
\end{aligned}
$$

with $N$ the number of pixels at the spatial resolution $x \mathrm{~km}$. Three-dimensional BTs at all scales are estimated by averaging the $50 \mathrm{~m} \mathrm{BT}$ to the $x \mathrm{~km}$ scale, while 1-D BTs are directly computed at the $x \mathrm{~km}$ scale at the averaged optical thickness. Because averaging BTs from a fine to a coarser spatial resolution will give a different result than BTs of the averaged optical thickness, we thus compared here how the nonlinearity between BT and optical thickness, as well as 3-D radiative effects, impacts TOA BTs at a given spatial resolution.

In order to separate the contribution of the PPHB and HRT to the total AMD, we also average the $50 \mathrm{~m}$ 1-D radiances to each $x \mathrm{~km}$ scale. The PPHB is then the arithmetic mean difference between averaged 1-D and non-averaged 1-D TOA BT viewed from nadir as a function of the spatial resolution, and is given by

$$
\begin{aligned}
& \operatorname{PPHB}\left(\overline{\Delta \mathrm{BT}_{x \mathrm{~km}}^{1-\mathrm{D}-1-\mathrm{D}}}\right)= \\
& {\left[\sum_{i=1}^{N}\left({\overline{\mathrm{BT}_{50 \mathrm{~m}}^{1-\mathrm{D}}}}^{x \mathrm{~km}}-\mathrm{BT}_{x \mathrm{~km}}^{1-\mathrm{D}}\right)\right] / N .}
\end{aligned}
$$

Note that, because the PPHB is always positive or nil, $\overline{\Delta \mathrm{BT}_{x \mathrm{~km}}^{1-\mathrm{D}-1-\mathrm{D}}}$ is also either positive or nil. It is straightforward to be positive for the whole field, but locally, on the scale of a pixel, it can be either positive or negative, contributing to increase or reduce the AMD.

To highlight the absolute effect of the HRT, which can be considered as the mean deviation of the BT due to HRT, we also calculate the total mean absolute difference (MAD) between averaged 3-D and non-averaged 1-D TOA BT viewed from nadir as a function of the spatial resolution using the following equation:

$$
\begin{aligned}
& \operatorname{MAD}\left(\overline{\left.\Delta \mathrm{BT}_{x \mathrm{~km}}^{3-\mathrm{D}-1-\mathrm{D}}\right)=}\right. \\
& {\left[\sum_{i=1}^{N}\left(\left|{\overline{\mathrm{BT}_{50 \mathrm{~m}}^{3-\mathrm{D}}}}^{x \mathrm{~km}}-\mathrm{BT}_{x \mathrm{~km}}^{1-\mathrm{D}}\right|\right)\right] / N .}
\end{aligned}
$$

This is almost the same as Eq. (2) but for the sum of the absolute value of the difference. The mean deviation due to HRT at each spatial resolution is then obtained by subtracting the PPHB from the total MAD ( $\mid$ HRT $\mid=$ MAD - PPHB). The MAD allows us to represent, at each spatial resolution, the mean deviation of the BT due to the cumulative effects of PPHB and |HRT|, and it is this parameter that we seek to minimize in order to estimate the optimal pixel size for IR cirrus retrievals.

Figure 8 shows the AMD and MAD in panel (a) and in panel (b) the PPHB and $|\mathrm{HRT}|$ of $\overline{\triangle \mathrm{BT}}$ estimated at TOA from nadir for the whole cirrus field as a function of the spatial resolution for the MODIS TIR channels centered at 8.52, 11.01, 12.03 and $13.36 \mu \mathrm{m}$. In Fig. 8, we note that, for all channels, AMD is always smaller than MAD because the PPHB can be partially offset by the HRT when it is negative.

As the behavior is different for different channels, we discuss first the more absorbing channels (centered at 11.01, 12.03 and $13.36 \mu \mathrm{m})$ and then the more scattering channel (centered at $8.52 \mu \mathrm{m}$ ). 

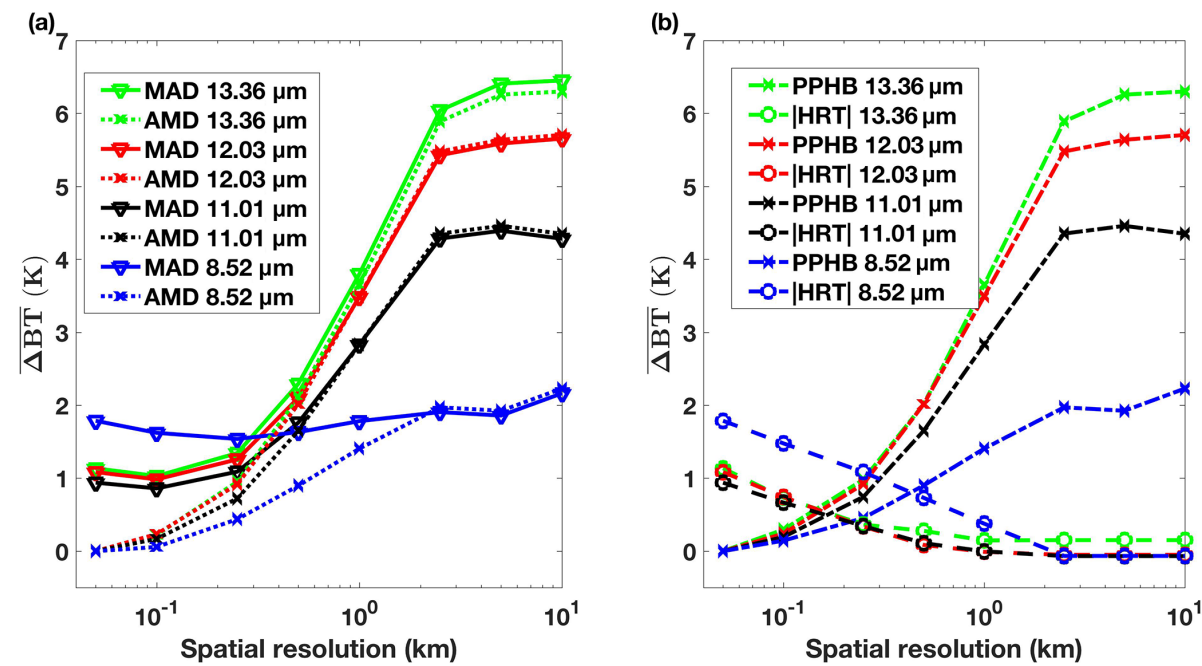

Figure 8. (a) Mean absolute difference (MAD) and arithmetic mean difference (AMD) between brightness temperatures computed in 3-D or 1-D following Eq. (2) and (b) plane-parallel and homogeneous bias (PPHB) and mean deviation due to the horizontal radiative transport $(|\mathrm{HRT}|)$ on brightness temperatures as a function of the spatial resolution for channels at 8.52, 11.01, 12.03 and $13.36 \mu \mathrm{m}$.

\subsection{Channels centered at 11.01, 12.03 and $13.36 \mu \mathrm{m}$}

In Fig. 8, it is evident that $\overline{\Delta \mathrm{BT}}$ at $11.01,12.03$ and $13.36 \mu \mathrm{m}$ plotted as a function of the spatial resolution have approximately the same behavior because the optical properties of the cirrus at these wavelengths are quite similar. As previously discussed, the largest heterogeneity bias for these channels is due to the PPHB (increasing with decreasing spatial resolution), leading to a maximum $\overline{\Delta \mathrm{BT}}$ for the coarsest spatial resolution. In our case, at the spatial resolution of $10 \mathrm{~km}$, the whole cirrus field is considered horizontally homogeneous, leading to the largest PPHB and AMD or MAD total biases. The differences between the three channels are due to the differences in cloud absorption. Considering the optical properties in Table 2, $\overline{\Delta \mathrm{BT}}$ increases with the absorption coefficient $\sigma_{\mathrm{a}}$. Indeed, the PPHB increases with cloud absorption (Fauchez et al., 2012, 2014) in the range where BT is a nonlinear function of optical thickness $\tau(0<\tau<10$ approximately). The larger AMD or MAD total biases are reached for the channel centered at $13.36 \mu \mathrm{m}$, with MAD of about $\overline{\Delta \mathrm{BT}}=6.5 \mathrm{~K}$ at $10 \mathrm{~km}$.

In fact, as the spatial resolution is improved from 10 to $2.5 \mathrm{~km}$, the $\overline{\Delta \mathrm{BT}}$ are quite stable as the field heterogeneity between these spatial resolutions is similar (i.e., the number of fallstreak in a $2.5 \mathrm{~km}$ box is similar to that of the whole field). Note that, at these scales, the AMD or MAD total biases and PPHB are close and the HRT error approaches 0 because the photon mean path is much shorter than these scales.

$\overline{\triangle \mathrm{BT}} \mathrm{AMD}$ and MAD drastically change below $2.5 \mathrm{~km}$ where the PPHB rapidly decreases with the improving spatial resolution. At $1 \mathrm{~km}$, we can see that the |HRT| effect (Fig. 8b) slightly increases, even though this is more clearly visible at $500 \mathrm{~m}$. Between 250 and $100 \mathrm{~m}$, the HRT curves cross the
PPHB curves and the HRT effect becomes the dominant effect. At $50 \mathrm{~m}$, the PPHB is nil because this is the same spatial resolution as that of the model. However, the $|\mathrm{HRT}|$ effect is the largest at $50 \mathrm{~m}$ because photons can easily propagate through many small $50 \mathrm{~m}$ pixels. It is important to note that the competition between the two effects leads to a minimum overall MAD around $100 \mathrm{~m}$ for these three channels.

\subsection{Channel centered at $8.52 \mu \mathrm{m}$}

The heterogeneity and horizontal transport effects on BT as functions of the spatial resolution have very different behavior at $8.52 \mu \mathrm{m}$ due to a stronger cloud scattering. Indeed, in this channel, the single-scattering albedo is 0.3 above the value for the three other channels (see Table 2). A stronger cloud scattering has two consequences: (i) a smaller PPHB due to a decrease in cloud absorption and emission for an equivalent extinction and (ii) a larger IPAE due to an increase in IHRTI. Indeed, we can see that, at $10 \mathrm{~km}, \overline{\Delta B T}$ is equal to $2.1 \mathrm{~K}$ instead of $4.2,5.8$ and $6.5 \mathrm{~K}$ for the channels centered at $11.01,12.03$ and $13.36 \mu \mathrm{m}$, respectively, implying that the PPHB is smaller. We can also see in Fig. 8 that, similar to the three other channels, $\overline{\triangle \mathrm{BT}} \mathrm{AMD}$ or MAD are almost constant from 10 to $2.5 \mathrm{~km}$ and the HRT effect is on average nil at these scales. However, below $2.5 \mathrm{~km}$, moving to higher spatial resolutions reduces the PPHB but increases $|\mathrm{HRT}|$ almost proportionally, leading to a relatively stable $\operatorname{MAD}(\overline{\Delta \mathrm{BT}})$. Nevertheless, we can see that the MAD minimum is located at about $250 \mathrm{~m}$ spatial resolution, which is a bit larger than for the three other channels because the stronger scattering effects and the weaker cloud absorption allow more photons to propagate farther at $8.52 \mu \mathrm{m}$. Note that the $\overline{\Delta \mathrm{BT}}$ values for the four channels are closer to each other for high than for coarse spatial resolutions. When the effects on BTs are 
(a)

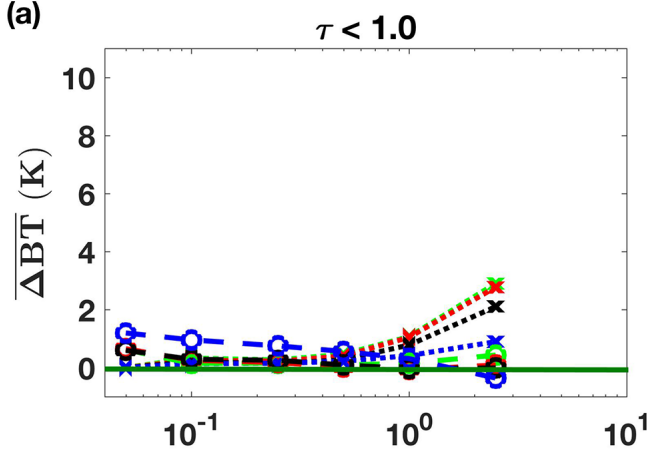

(c)

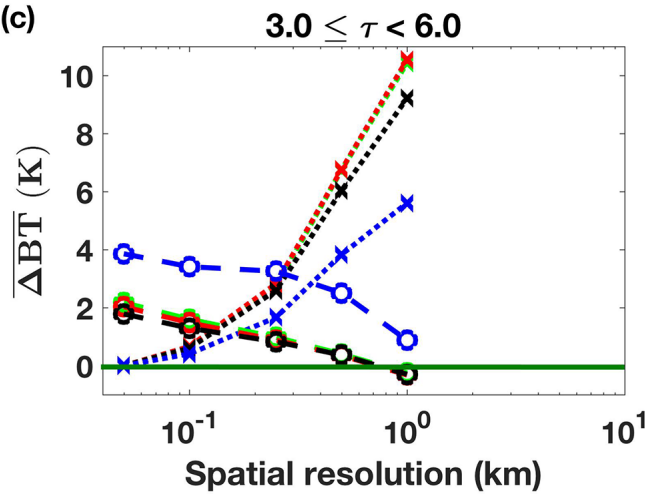

$1.0<\tau<3.0$

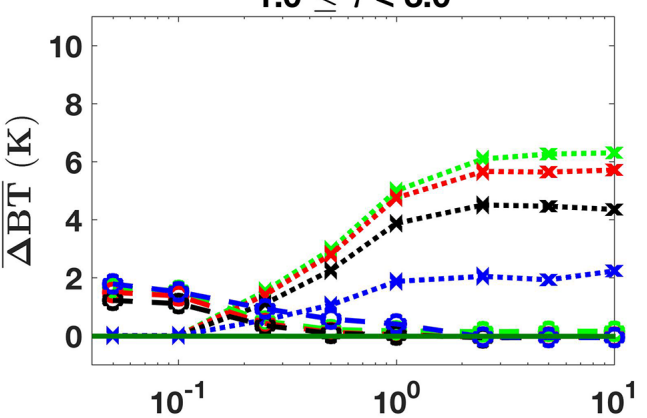

$\tau>6.0$

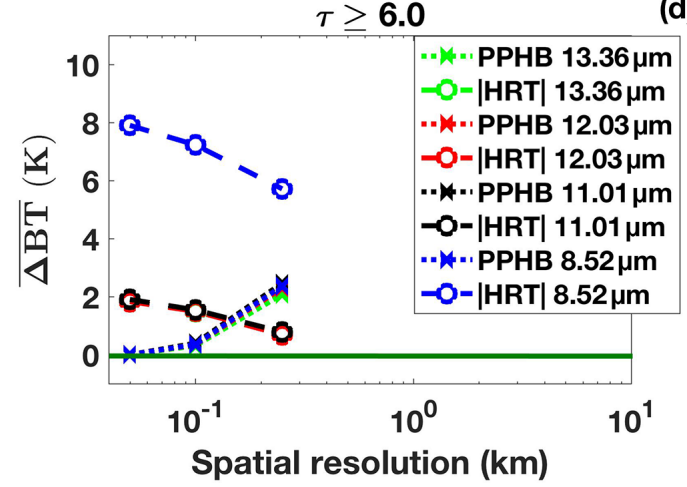

Figure 9. Scene-average, plane-parallel and homogeneous bias (PPHB) and mean deviation due to the horizontal radiative transport (|HRT $\mid$ ) effect on brightness temperatures $(\overline{\Delta \mathrm{BT}})$ for (a) small, (b) medium, (c) large and (d) very large pixel optical thicknesses as a function of spatial resolution in channels centered at 8.52, 11.01, 12.03 and $13.36 \mu \mathrm{m}$. The small optical thickness range corresponds to 28735 pixels, the 17305 medium pixels, the 5028 large pixels and the 1063 very large pixels.

roughly the same for all channels, the $\operatorname{MAD}(\overline{\Delta \mathrm{BT}})$ impact on retrieved products may be mitigated (not shown here). Note that these differences are dependent on the CED, for which the single-scattering albedo varies with wavelength. For very large CED $(>80 \mu \mathrm{m})$ the single scattering varies less with wavelength (about the value of $\mathrm{CED}=20 \mu \mathrm{m}$ for $13.36 \mu \mathrm{m})$, reducing differences between channels and therefore the overall impact in the retrieval.

To summarize, for this cirrus field, the best resolution for mitigating the cumulative effect of the PPHB and horizontal transport effect is about $100 \mathrm{~m}$ for the three channels with stronger cloud absorption and is about $250 \mathrm{~m}$ for the channel centered at $8.52 \mu \mathrm{m}$.

By a quick and simple sensitivity study, we can simulate the inhomogeneity impact for various average cloud properties by sampling the whole cloud scene according to three important parameters:

- the brightness temperature contrast between clear sky and opaque cloudy pixels

- the average cloud optical thickness

- the average cloud heterogeneity.

The increase in the brightness temperature contrast between clear sky and opaque cloudy pixels $(\triangle \mathrm{BT}(\mathrm{CS}-\mathrm{OP}))$ noticeably increases the PPHB (larger nonlinear BT vs. $\tau$ averaging effect) as well as the IPAE (HRT has a larger impact if columns have more different opacities). For example, Fauchez et al. (2014) has shown in their Fig. 16 that when increasing the cirrus top altitude from about $8 \mathrm{~km}$ to about $11 \mathrm{~km}$, the total effect (AMD) on BT is multiplied by 3 for the channel centered at about $8 \mu \mathrm{m}$ and about 2.5 for the channels around 10 and $12 \mu \mathrm{m}$. These factors will of course depend on the cloud opacity and surface and atmospheric temperature. However, the resolution of the minimum heterogeneity effect should not be affected by a change in $\triangle \mathrm{BT}(\mathrm{CS}-\mathrm{OP})$ since only the photon energy will change but not its mean path. Considering the computational times involved, we chose to rely on this hypothesis and not do other time-consuming runs.

However, the impact of changing the two other parameters is that the average cloud optical thickness and heterogeneity can be more easily tested by sampling the cloud pixels in different optical thickness or heterogeneity ranges. Indeed, the $50,100,250,500,1,2.5,5$ and $10 \mathrm{~km}$ spatial resolutions correspond to $40000,10000,1600,400,100,40,16$ and 1 pixels, respectively, which represent a large number of pixels with various optical thicknesses and heterogeneities. 
(a)

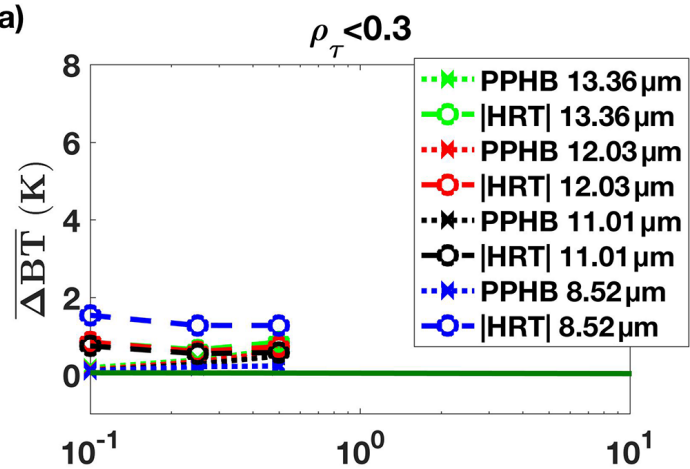

(b)

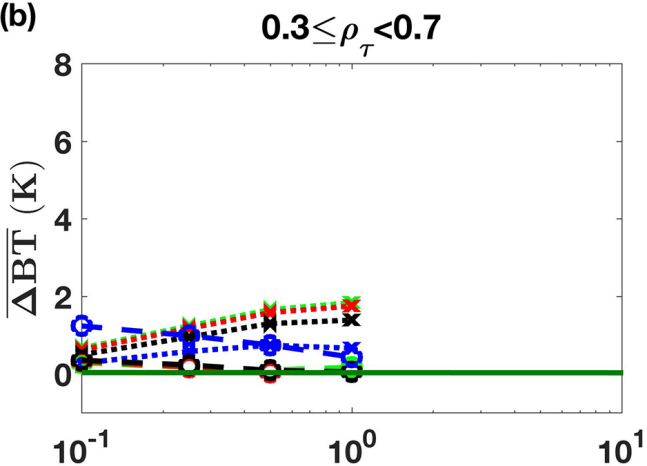

(c)

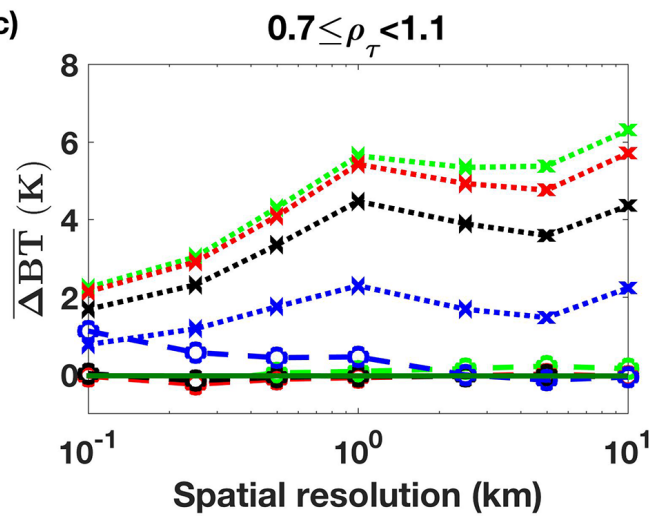

(d)

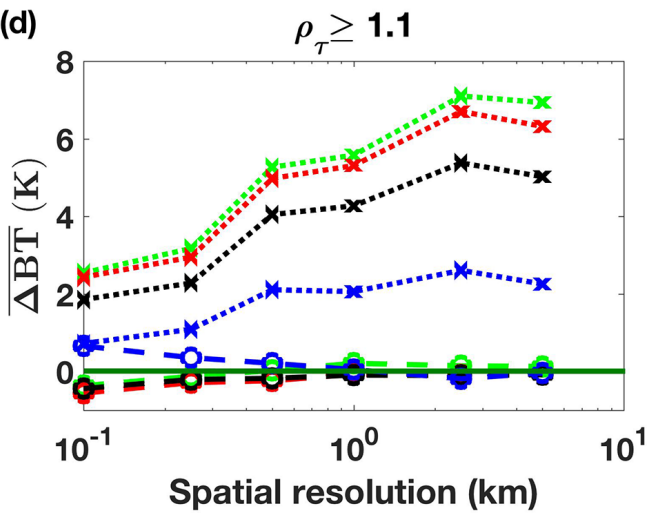

Figure 10. Scene-average, plane-parallel and homogeneous bias (PPHB) and mean deviation due to the horizontal radiative transport (|HRT|) effect on brightness temperatures $(\overline{\Delta \mathrm{BT}})$ for (a) small, (b) medium, (c) large and (d) very large pixel inhomogeneity $\left(\rho_{\tau}\right)$ as a function of spatial resolution in channels centered at $8.52,11.01,12.03$ and $13.36 \mu \mathrm{m}$. The small optical thickness range corresponds to 8969 pixels, the 2724 medium pixels, the 347 large pixels and the 89 very large pixels.

For every spatial resolution, we sampled pixels in four optical thickness $\tau$ (at $12.03 \mu \mathrm{m}$ ) categories:

- small optical thicknesses: $\tau<1.0$ (28735 pixels)

- medium optical thicknesses: $1.0 \leqslant \tau<3.0$ (17305 pixels)

- large optical thicknesses: $3.0 \leqslant \tau<6.0$ (5028 pixels)

- very large optical thicknesses: $\tau \geqslant 6$ (1089 pixels).

Similarly, the optical thickness heterogeneity parameter $\rho_{\tau}=[\operatorname{SD}[\tau] /<\tau>]$ (Szczap et al., 2000) is also sampled in four ranges:

- small optical thickness heterogeneity: $\rho_{\tau}<0.3$ (8969 pixels)

- medium optical thicknesses heterogeneity: $0.3 \leqslant \rho_{\tau}<$ 0.7 (2724 pixels)

- large optical thicknesses heterogeneity: $0.7 \leqslant \rho_{\tau}<1.1$ (347 pixels)

- very large optical thicknesses heterogeneity: $\rho_{\tau} \geqslant 1.1$ (117 pixels).
The results of this sensitivity study are presented in Fig. 9 for the optical thickness and Fig. 10 for the heterogeneity of the optical thickness, and the results are summarized in Table 4. In this table, we can see the spatial resolutions at which PPHB is larger than the $\mid$ HRT $\mid$ effect, and vice versa, as well as the minimum of the total MAD effect. For clarity we chose not to show the AMD and MAD values in the figures, and to keep only the MAD values in the table. We can see that the change in the optical thickness or the heterogeneity deeply affects the relative strength of the PPHB and $\mid$ HRT $\mid$. As previously seen, the PPHB dominates at large scales, while the $|\mathrm{HRT}|$ dominates at small scales, except for $\tau \geqslant 6$. Indeed, the PPHB increases with the optical thickness while |HRT| decreases because of the increase in the cloud absorption. When the heterogeneity $\rho_{\tau}$ increases, this allows the PPHB to increase and to be larger than the $|\mathrm{HRT}|$ even at finer spatial resolutions (roughly shifted from 250 to $100 \mathrm{~m}$ between $\rho_{\tau}<0.3$ and $\rho_{\tau} \geqslant 1.1$ ). Indeed, increasing $\rho_{\tau}$ leads, on average, to an increase in the optical thickness and thus the cloud absorption which enhances the PPHB but mitigates the $|\mathrm{HRT}|$. In addition, we can see that the spatial resolution at which the MAD is minimum is quite stable. Most of the time, this is at $100 \mathrm{~m}$ spatial resolution, followed by 


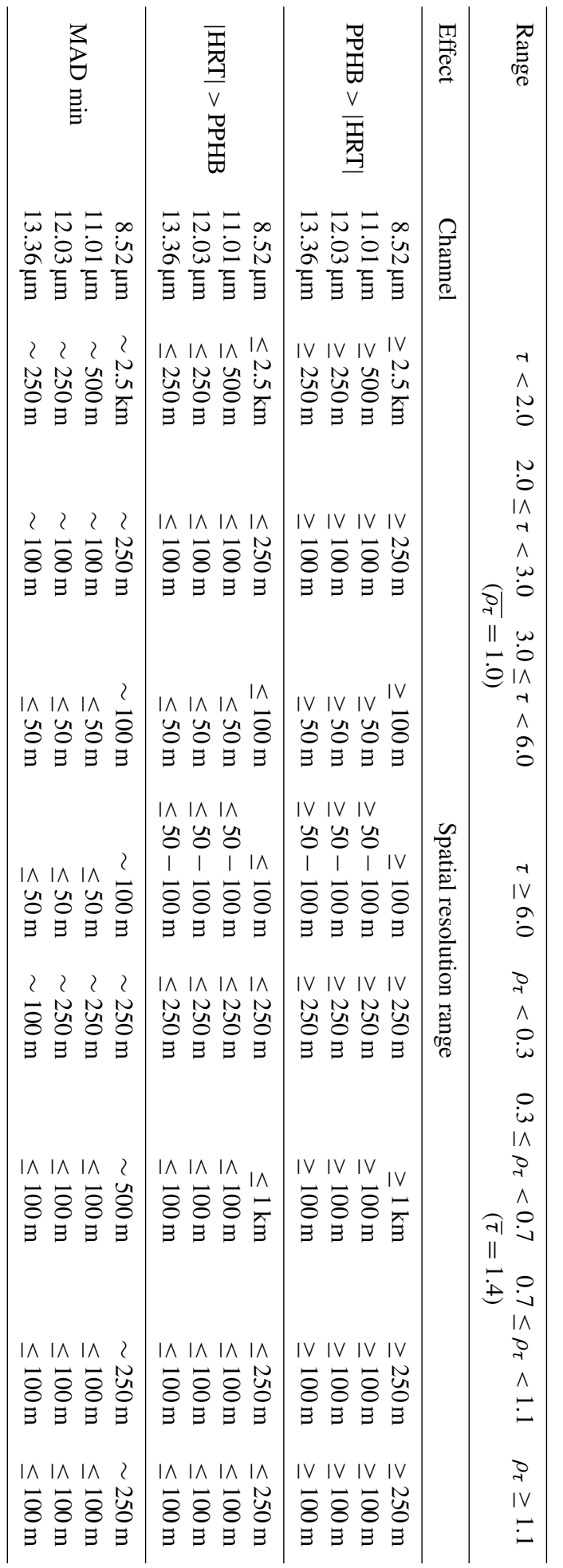

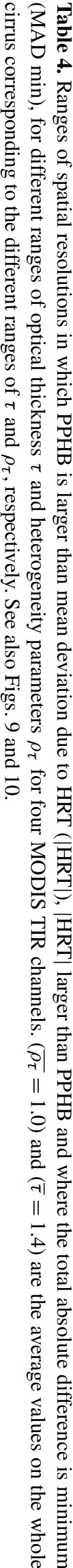

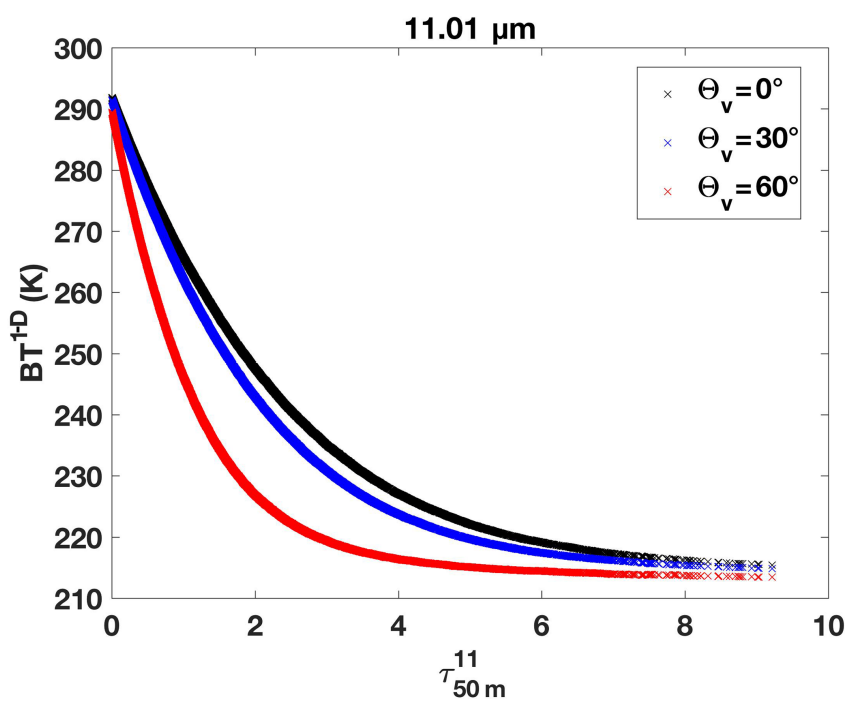

Figure 11. One-dimensional brightness temperatures as a function of the $50 \mathrm{~m}$ optical thickness $\tau_{50 \mathrm{~m}}^{1-\mathrm{D}}$ for viewing zenith angles $\Theta_{\mathrm{V}}=$ 0,30 and $60^{\circ}$ at a constant view azimuth angle of $0^{\circ}$.

$250 \mathrm{~m}$ and $50 \mathrm{~m}$. For $50 \mathrm{~m}$ no clear conclusions can be drawn because this is the smallest scale of the simulation. These conclusions are consistent with those of Fig. 8 for the whole cirrus field. Note that in Figs. 9 and 10, the IHRTI can be negative in one specific optical thickness or heterogeneity range since this is on the whole field, where the HRT is, on average, close to nil.

\section{Heterogeneity effects as functions of the observation scale for off-nadir views}

In the previous sections, results were shown for simulated observations from nadir. In this section, we discuss off-nadir observation geometries. In addition to the PPHB and HRT effects, another bias appears when looking off-nadir. Indeed, the oblique line of sight can cross many different cloudy columns in 3-D radiative transfer mode, while in 1-D, the cloudy column underneath a given pixel is considered horizontally infinite and thus fully containing the line of sight. We name this last bias the tilted homogeneous extinction assumption bias (THEAB). Note that the results of this section are strongly dependent on the cloud structure (with fallstreaks or not) and may be generalized to cirrus with similar patterns.

First of all, we can see in Fig. 11 that for off-nadir views, the PPHB is enhanced due to the increase in the curvature (non linearity) between BT and optical thickness with the view zenith angle. Note that we can also see in this figure that the saturation in BT with respect to changes in optical thickness appears earlier at $\Theta_{\mathrm{v}}=60^{\circ}\left(\tau_{50 \mathrm{~m}}^{1-\mathrm{D}} \sim 7\right)$ than at $\Theta_{\mathrm{v}}=30^{\circ}$ $\left(\tau_{50 \mathrm{~m}}^{1-\mathrm{D}} \sim 8\right)$ and $0^{\circ}\left(\tau_{50 \mathrm{~m}}^{1-\mathrm{D}} \sim 9\right)$. 
(a)

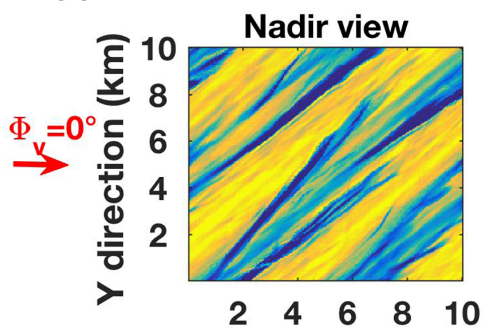

3-D

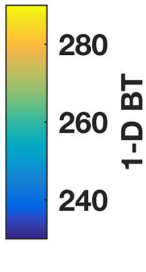

(b)

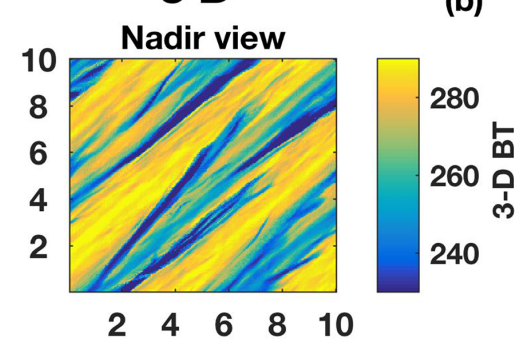

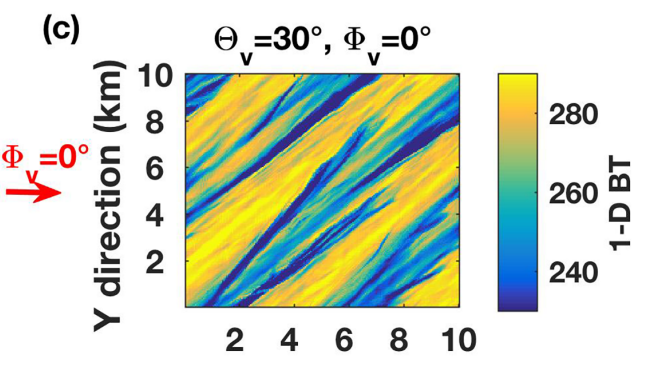

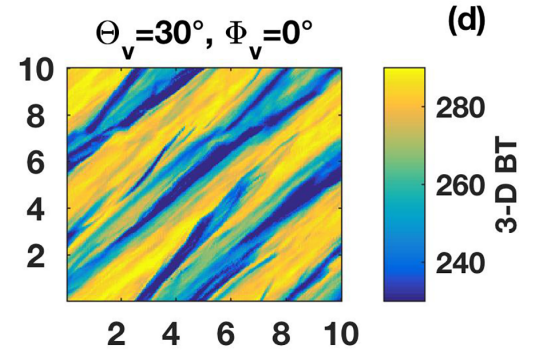

(e)
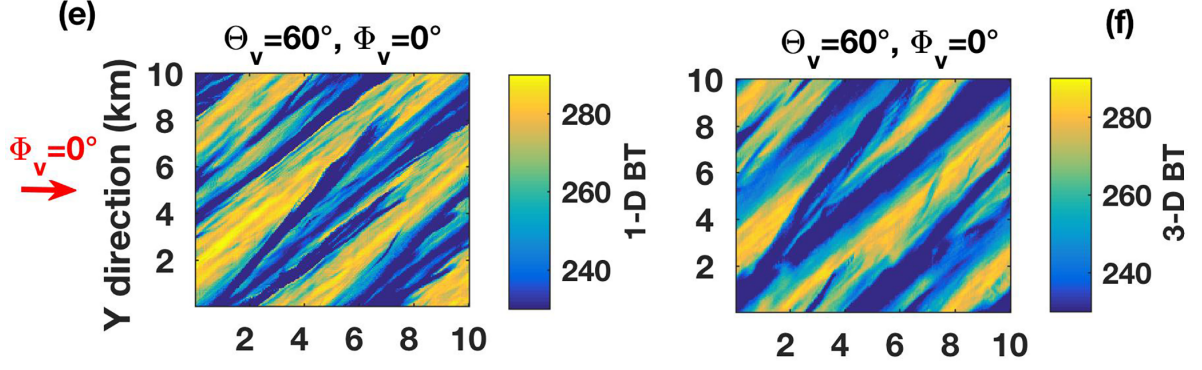

(g)

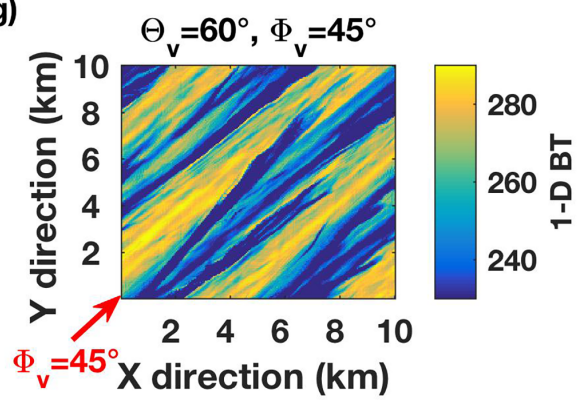

Figure 12. One-dimensional (a, c, e, g) and three-dimensional $(\mathbf{b}, \mathbf{d}, \mathbf{f}, \mathbf{h}) \mathrm{BT}$ fields at $11.01 \mu \mathrm{m}$ and at $50 \mathrm{~m}$ spatial resolution view at a zenith angle of $\Theta_{\mathrm{v}}=0,30$ and $60^{\circ}$, respectively, for an azimuth viewing angle of $\Phi_{\mathrm{V}}=0^{\circ}$ and $\Phi_{\mathrm{V}}=45^{\circ}$ represented by the black arrows.

BTs differences between the viewing geometries can be seen in Fig. 12 with 1-D BT (left column) and 3-D BT (right column). In 1-D we can clearly see that increasing the viewing zenith angle reduces the average BT and that small differences appear for different azimuth view angles. In 3-D, because the line of sight can cross many different cloudy columns, the radiative field is much more dependent on both the zenith and azimuth view angles. The differences between the 1-D and 3-D fields for oblique views is mostly due to the THEAB.
Like the HRT effect, the THEAB is due to the IPAE and both effects are thus merged and represented in Fig. 13 by the IPAE. In Fig. 13 we can see the AMD (bold lines with squares), the MAD (bold lines with triangles), the PPHB (dashed line with crosses) and the IPAE (dotted line with stars) of $(\overline{\triangle \mathrm{BT}})$ in panel (a) for viewing zenith angles $\Theta_{\mathrm{v}}=$ $0^{\circ}, 30^{\circ}$ and $60^{\circ}$ at a viewing azimuth angle of $\Phi_{\mathrm{v}}=0^{\circ}$, and panels (b) and (c) for viewing azimuth angles of $\Phi_{\mathrm{v}}=0^{\circ}$, $45^{\circ}, 90^{\circ}$ and $180^{\circ}$ at $\Theta_{\mathrm{v}}=30^{\circ}$ and $60^{\circ}$ as a function of the spatial resolution for the channel centered at $11.01 \mu \mathrm{m}$ only. In Fig. 13a, $\operatorname{MAD}(\overline{\Delta B T})$ at $\Theta_{v}=30^{\circ}$ and $60^{\circ}$ for spatial res- 
(a) Constant view azimuth angle $\Phi_{\mathrm{v}}=0^{\circ}$

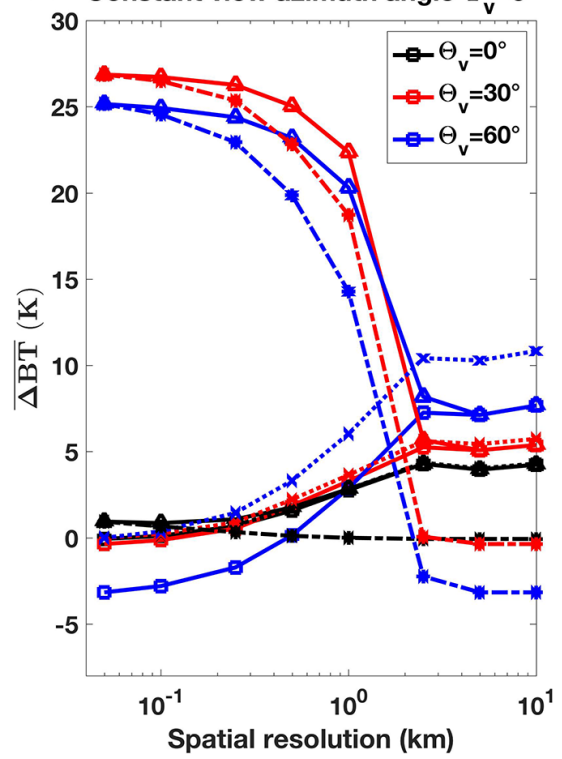

(b) Constant view zenith angle $\Theta_{v}=30^{\circ}$

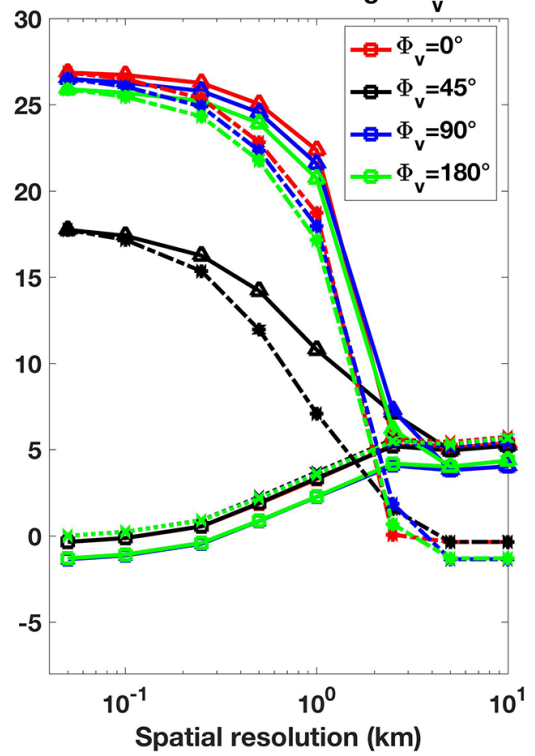

(c) Constant view zenith angle $\Theta_{v}=60^{\circ}$

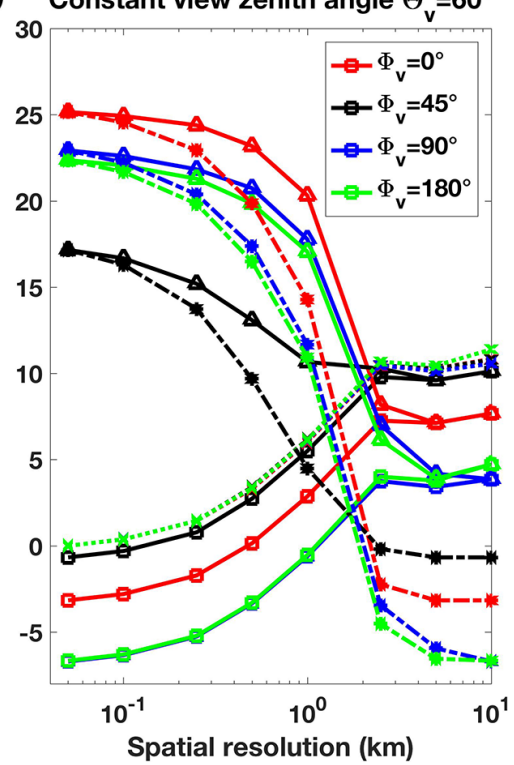

Figure 13. Mean absolute difference (MAD, lines with triangles) and average mean difference (AMD, lines with squares) between 3-D and 1-D brightness temperatures estimated following Eq. (2), plane-parallel and homogenous bias (PPHB, doted lines with crosses) and independent pixel approximation error (IPAE, dashed lines with stars) as a function of the spatial resolution for the channel centered at $11.01 \mu \mathrm{m}$ and as a function of (a) the viewing zenith angle $\Theta_{\mathrm{V}}$ at an azimuth angle of $\Phi_{\mathrm{V}}=0^{\circ}$, (b) the viewing azimuth angle at $\Theta_{\mathrm{V}}=30^{\circ}$ and (c) the viewing azimuth angle at $\Theta_{\mathrm{v}}=60^{\circ}$.

olutions between 1 and $10 \mathrm{~km}$ are larger than at nadir because of the larger PPHB as seen in Fig. 11.

In Fig. 13a, the mean absolute difference $\operatorname{MAD}(\overline{\Delta \mathrm{BT}})$ at $\Theta_{\mathrm{v}}=30^{\circ}$ and $60^{\circ}$ is very large below $\sim 1 \mathrm{~km}$ due to the fact that the line of sight crosses many different columns in 3D (large THEAB, which contributes strongly to the IPAE represented by the dashed lines with stars). However, when looking at the arithmetic mean difference $\operatorname{AMD}(\overline{\Delta \mathrm{BT}})$, we see that at $\Theta_{\mathrm{v}}=60^{\circ}$, it is negative for spatial resolutions below $500 \mathrm{~m}$ due to two effects: (i) first, in 3-D, due to the very oblique view, the line of sight crosses many cloudy columns of various optical properties for which the extinction is summed and leads, in most cases, to large optical paths. Such large optical paths imply that the top of the cirrus mostly contributes to the TOA BT. In contrast, some lines of sight cross small optical thicknesses at which photons emitted from the surface, warmer than the cloud, contribute to the TOA BT. This leads to 3-D BT being smaller than 1-D $\mathrm{BT}$ on average, and thus to negative $\operatorname{AMD}(\overline{\Delta \mathrm{BT}})$ values. We can also see this in Fig. 12f where the blue color (cold emission temperature at the cloud top) is more present than in 1-D (Fig. 12e). (ii) In 3-D, the line of sight crosses so many different columns that the difference between nearby lines of sight is reduced and the heterogeneity of the BT field is smaller in $3-\mathrm{D}(\mathrm{SD}[\mathrm{BT}] \sim 20.2 \mathrm{~K})$ than in $1-\mathrm{D}(\mathrm{SD}[\mathrm{BT}] \sim 22.3 \mathrm{~K})$ as we can see in Fig. $12 \mathrm{f}$ by comparison to Fig. 12e, respectively. Then, when $50 \mathrm{~m}$ BT values are averaged following Eq. (2), 1-D BTs are increased more by the PPHB than 3-
D BTs are, which contributes to the overall tendency of 1-D $\mathrm{BT}>3-\mathrm{D}$ BT and to the negative value of $\operatorname{AMD}(\overline{\Delta \mathrm{BT}})$.

While both effects are particularly strong below $500 \mathrm{~m}$ where the pixel size is small, they occur at every spatial resolution, explaining why $\mathrm{PPHB}$ is always larger than the AMD due to the IPAE. Note that, contrary to the PPHB, the THEAB contribution to the IPAE does not increase monotonously with $\Theta_{\mathrm{v}}$ because it is related to the heterogeneity of the extinction along the line of sight for 3-D computations at $50 \mathrm{~m}$, which can be smaller at $\Theta_{\mathrm{v}}=60^{\circ}$ than at $\Theta_{\mathrm{v}}=30^{\circ}$. We can also see that the IPAE is negative and the AMD is positive at $2.5,5$ and $10 \mathrm{~km}$ at $\Theta_{\mathrm{v}}=60^{\circ}$. Knowing that the HRT effect does not impact BT because the pixel size is too large, the IPAE is essentially due to the THEAB. In contrast to the higher spatial resolutions, the number of cloudy columns crossed by the line of sight is small and the large averaging homogenizes the field and thus reduces the AMD to a level close to or equal to the MAD. As a result, the AMD is smaller than the PPHB, which means that at coarse spatial resolutions the PPHB clearly dominates and the AMD is reduced, and not amplified, by the IPAE. Since $\mathrm{AMD}=\mathrm{PPHB}+\mathrm{IPAE}$, the IPAE is negative as PPHB is larger than AMD or MAD. For $\Theta_{\mathrm{V}}=60^{\circ}$, the conclusions are similar to those for $\Theta_{\mathrm{V}}=30^{\circ}$, but with larger differences due to the greater IPAE between 3-D and 1-D BTs.

Concerning the change of the viewing azimuth angle at $\Theta_{\mathrm{v}}=30^{\circ}$ and $60^{\circ}$, the difference in AMD, MA, PPHB and IPAE between the four angles is quite small except at $\Phi_{\mathrm{V}}=$ 
$45^{\circ}$. Indeed, at this azimuth angle, the line of sight is parallel to the cirrus fallstreaks as we can see in Fig. $12 \mathrm{~g}$ and $\mathrm{h}$ for $\Theta_{\mathrm{v}}=60^{\circ}$. Therefore, the variability along the oblique line of sight is weaker, reducing the smoothing effect of the 3-D field, which is closer to the 1-D field averaged heterogeneity ( $\mathrm{SD}[\mathrm{BT}] \sim 21.1 \mathrm{~K}$ in 3 -D and $\mathrm{SD}[\mathrm{BT}] \sim 22.3 \mathrm{~K})$. In addition, the line of sight can pass only optically small paths and result in large BTs just as in 1-D. As a result, $\operatorname{MAD}(\overline{\Delta B T})$ at $\Phi_{\mathrm{v}}=45^{\circ}$ is reduced at spatial resolutions at which fallstreaks are still observable $(\leq 2.5 \mathrm{~km})$. Above this value, the spatial resolution is so low that the fallstreaks are smoothed and the effect disappears.

Off-nadir, it is not easy to determine the spatial resolution at which the absolute value of $\overline{\Delta \mathrm{BT}}$ occurs due to the combined heterogeneity and 3-D effects reaching a minimum because the spatial resolution depends on the viewing angle as well as on horizontal and vertical inhomogeneity. However, looking at Fig. 13 we can say that this location is at a coarser resolution than at nadir, as the THEAB drastically increases the $\overline{\triangle \mathrm{BT}}$, especially at high spatial resolutions. The spatial resolution at nadir where the AMD of $\overline{\Delta B T}$ is the most mitigated for nadir view therefore sets a lower limit for off-nadir viewing geometries on both the AMD of $\overline{\Delta B T}$ and the spatial resolution at which the combined effects are minimum. These results were limited to the channel centered at $11.01 \mu \mathrm{m}$ because computations for other channels were too computationally expensive. However, optical properties for channels at $11.01,12.03$ and $13.36 \mu \mathrm{m}$ are close, leading to similar $\operatorname{MAD}(\overline{\Delta \mathrm{BT}})$ for nadir view as seen in Fig. 8. $\operatorname{MAD}(\overline{\Delta \mathrm{BT}})$ for other view angles should therefore be equivalent to the one at $11.01 \mu \mathrm{m}$. Only the $8.52 \mu \mathrm{m}$ channel may have a different behavior. However, considering $\operatorname{MAD}(\overline{\Delta B T})$ differences between 11.01 and $8.52 \mu \mathrm{m}$ in Fig. 8, we can expect that $\operatorname{MAD}(\overline{\Delta \mathrm{BT}})$ for $8.52 \mu \mathrm{m}$ will be larger for a smaller pixel size due to the larger scattering and the greater horizontal radiative transport.

\section{Conclusions}

The accurate remote sensing of cirrus clouds is very important in order to improve the parametrization of clouds in climate models and to better understand their role in the Earthatmosphere system. Cloud heterogeneities may have a significant impact on the accuracy of retrieved cloud optical properties. In this work, we model the impact of cirrus cloud heterogeneities on top-of-the-atmosphere brightness temperatures as a function of the spatial resolution from $50 \mathrm{~m}$ to 10 $\mathrm{km}$ and at four MODIS thermal infrared channels centered at $8.52,11.01,12.03$ and $13.36 \mu \mathrm{m}$. A three-dimensional cirrus cloud structure is modeled with the 3DCLOUD cloud generator and radiative transfer simulations are performed with the 3DMCPOL code. In this study, we assume that TOA brightness temperature differences between $\mathrm{BT}$ computation assuming 1-D RT inside a homogeneous pixel and 3-D RT in- side a heterogeneous pixel depend on two effects: (i) the optical thickness horizontal inhomogeneity leading to the planeparallel approximation bias and the (ii) horizontal radiative transport effect due to the independent pixel approximation error. The cloud vertical heterogeneities of optical properties are neglected here, based on the findings of Fauchez et al. (2014, 2015). As previous studies already showed, the PPHB is the larger heterogeneity effect for nadir observations at the typical spatial resolution of polar orbiters such as AIRS, MODIS or IIR. The PPHB impacts mainly the low spatial resolutions, while the IPAE impacts mainly the high spatial resolutions. Although, due to the IPAE, the amplitude of the error in BT can be large at high spatial resolutions, the difference between the errors for different channels is quite small in comparison to the difference at coarse resolution. A similar error between channels can then mitigate the impact on the optical property retrieval. For our simulated cirrus case, we find that the approximate spatial resolution at which the PPHB and HRT effects lead to a minimum total effect at nadir is between 100 and $250 \mathrm{~m}$. In order to extrapolate this result to different cirrus clouds, a sensitivity study has been conducted. The results show that changing the average cloud optical thickness affects the magnitude of the effects but does not significantly change the spatial resolution of the minimum. A spaceborne radiometer with a nadir spatial resolution between 100 and $250 \mathrm{~m}$ will allow the retrieval of cirrus optical properties in the thermal infrared with mitigated overall heterogeneity and radiative effects. In future studies, we will investigate how the errors on COT and CED retrievals due to horizontal inhomogeneities and 3-D effects are scale dependent.

Concerning off-nadir views, when $\Theta_{\mathrm{v}}>0^{\circ}$, the line of sight may cross several different cloudy columns in 3-D RT but not in 1-D RT, leading to the tilted homogeneous extinction assumption bias. This strongly increases the mean deviation between 3-D and 1-D BT, especially at fine spatial resolutions. However, on average, an increase in viewing zenith angle decreases the 3-D BT values as well as their heterogeneity, reducing the total error due to PPHB and IPAE. The dependence of the total effect on the azimuth angle could also be important for particular viewing orientations with respect to the cloud. For instance, the cloud heterogeneity, and thus the total effect, is smaller when the line of sight is parallel to the fallstreaks of the cloud, and is larger elsewhere. It thus seems that, for arithmetic field average values, the minimum total effect arises at nadir. Also, the THEAB leads to a shift in the spatial resolution of the minimum total effect toward coarser spatial resolutions. Off-nadir, it is clear that the horizontal and vertical structure of the cloud may change the conclusions. However, we have chosen the uncinus cirrus structure (with fallstreaks corresponding to intervals of thick and thin optical thicknesses), which is one of the most common among the variety of cirrus structures. We can thus extrapolate that results may be comparable to other uncinus 
cirrus, but may be different from others structures such as the patchy structures of cirrus floccus.

Note that these simulations were performed for a unique CED of $20 \mu \mathrm{m}$, very common in cirrus clouds but relatively small. However, for example, increasing CED to $80 \mu \mathrm{m}$ leads to a convergence of the single-scattering albedo across all the TIR channels towards values between 0.5 and 0.6 ( 0.5 being the geometric optics limit). This implies less scattering and thereby horizontal transport in the $8.52 \mu \mathrm{m}$ channel $\left(\varpi_{0} \sim\right.$ 0.75 for $\mathrm{CED}=20 \mu \mathrm{m}$ in this study). The differences between channels should thus be weaker and consequently the impacts on cloud optical property retrievals, which depend on the radiance relative difference between channels. Also, because single-scattering albedo values for all the channels at $D_{\text {eff }}=80 \mu \mathrm{m}$ are close to those at $13.36 \mu \mathrm{m}$ for $D_{\text {eff }}=20 \mu \mathrm{m}$ used in this study, all the channels for $D_{\text {eff }}=80 \mu \mathrm{m}$ will have a similar heterogeneity effect on TOA BT across spatial resolutions than for the $13.36 \mu \mathrm{m}$ channel presented in this study. In Part 2 of this work we will study the impact of cirrus heterogeneities on visible and near-infrared MODIS channels and will make comparisons with the results of Part 1 here. We anticipate that the results will be different since 3-D effects are stronger for visible and near-infrared wavelengths and that solar geometries will play an important role. Additional perspectives will concern the impact of cirrus cloud heterogeneities on the optical property retrievals. Indeed, the dependence of heterogeneity and 3-D effects on the wavelength can be an issue for retrieval techniques using a combination of many wavelength ranges (such as optimal estimation methods). Other clouds, such as cumulus or stratocumulus, should also be considered because results are expected to be strongly dependent on the cloud type.

Code availability. The simulated data used in this study were generated by the 3DCLOUD (Szczap et al., 2014) and 3DMCPOL (Cornet et al., 2010; Fauchez et al., 2014) closed-source codes. Please contact the authors for more information.

Competing interests. The authors declare that they have no conflict of interest.

Acknowledgements. The authors acknowledge the Universities Space Research Association (USRA) through the NASA Postdoctoral Program (NPP) for their financial support. We thank Zhibo Zhang and the UMBC High Performance Computing Facility (HPCF) for the use of their computational resources (MAYA). The facility is supported by the US National Science Foundation through the MRI program (grant nos. CNS-0821258 and CNS-1228778) and the SCREMS program (grant no. DMS0821311), with additional substantial support from the University of Maryland, Baltimore County (UMBC). See www.umbc.edu/hpcf for more information on HPCF and the projects using its resources. We also thank the NASA Center for Climate Simulation (NCCS) for the use of their computational resources (Discover). We also gratefully acknowledge the two anonymous reviewers, who contributed their very relevant comments and improved the quality of the paper.

Edited by: Johannes Quaas

Reviewed by: two anonymous referees

\section{References}

Benassi, A., Szczap, F., Davis, A., Masbou, M., Cornet, C., and Bleuyard, P.: Thermal radiative fluxes through inhomogeneous cloud fields: a ensitivity study using a new stochastic cloud generator, Atmos. Res., 72, 291-315, 2004.

Buschmann, N., McFarquhar, G., and Heymsfield, A.: Effects of observed horizontal inhomogeneities within cirrus clouds on solar radiative transfer, J. Geophys. Res.-Atmos., 107, 4445, https://doi.org/10.1029/2001JD001273, 2002.

Cahalan, R. F. and Snider, J. B.: Marine stratocumulus structure, Remote Sens. Environ., 28, 95-107, 1989.

Cahalan, R. F., Ridgway, W., Wiscombe, W. J., Bell, T. L., and Snider, J. B.: The Albedo of Fractal Stratocumulus Clouds, J. Atmos. Sci., 5, 2434-2455, 1994.

Carlin, B., Fu, Q., Lohmann, U., Mace, J., Sassen, K., and Comstock, J. M.: High cloud horizontal inhomogeneity and solar albedo bias, J. Climate, 15, 2321-2339, 2002.

Chen, Y. and Liou, K. N.: A Monte Carlo method for 3D thermal infrared radiative transfer, J. Quant. Spectrosc. Ra., 101, 166178, 2006.

Choi, Y.-S. and Ho, C.-H.: Radiative effect of cirrus with different optical properties over the tropics in MODIS and CERES observations, Geophys. Res. Lett., 33, L21811, https://doi.org/10.1029/2006GL027403, 2006.

Cooper, S. J. and Garrett, T. J.: Identification of Small Ice Cloud Particles Using Passive Radiometric Observations, J. Appl. Meteorol. Clim., 49, 2334-2347, 2010.

Cooper, S. J., L'Ecuyer, T. S., Gabriel, P., Baran, A. J., and Stephens, G. L.: Performance assessment of a five-channel estimation-based ice cloud retrieval scheme for use over the global oceans, J. Geophys. Res.-Atmos., 112, D04207, https://doi.org/10.1029/2006JD007122, 2007.

Cornet, C., C-Labonnote, L., and Szczap, F.: Three-dimensional polarized Monte Carlo atmospheric radiative transfer model (3DMCPOL): 3D effects on polarized visible reflectances of a cirrus cloud, J. Quant. Spectrosc. Ra., 111, 174-186, https://doi.org/10.1016/j.jqsrt.2009.06.013, 2010.

Corti, T. and Peter, T.: A simple model for cloud radiative forcing, Atmos. Chem. Phys., 9, 5751-5758, https://doi.org/10.5194/acp9-5751-2009, 2009.

Davis, A., Marshak, A., Wiscombe, W., and Cahalan, R.: Multifractal characterizations of nonstationarity and intermittency in geophysical fields: Observed, retrieved, or simulated, J. Geophys. Res.-Atmos., 99, 8055-8072, 1994.

Davis, A., Marshak, A., Wiscombe, W., and Cahalan, R.: Scale Invariance of Liquid Water Distributions in Marine Stratocumulus - Part I: Spectral Properties and Stationarity Issues, J. Atmos. Sci., 53, 1538-1558, 1996. 
Davis, A., Marshak, A., Cahalan, R., and Wiscombe, W.: The Landsat Scale Break in Stratocumulus as a Three-Dimensional Radiative Transfer Effect: Implications for Cloud Remote Sensing, J. Atmos. Sci., 54, 241-260, 1997.

Dowling, D. R. and Radke, L. F.: A Summary of the Physical Properties of Cirrus Clouds, J. Appl. Meteorol., 29, 970-978, 1990.

Emde, C., Buras, R., and Mayer, B.: ALIS: An efficient method to compute high spectral resolution polarized solar radiances using the Monte Carlo approach, J. Quant. Spectrosc. Ra., 112, 16221631, 2011.

Fauchez, T., Cornet, C., Szczap, F., and Dubuisson, P.: Assessment of cloud heterogeneities effects on brightness temperatures simulated with a 3D Monte-Carlo code in the thermal infrared, International Radiation Symposium proceeding, Berlin, Germany, 4 pp., 2012.

Fauchez, T., Cornet, C., Szczap, F., Dubuisson, P., and Rosambert, T.: Impact of cirrus clouds heterogeneities on top-of-atmosphere thermal infrared radiation, Atmos. Chem. Phys., 14, 5599-5615, https://doi.org/10.5194/acp-14-5599-2014, 2014.

Fauchez, T., Dubuisson, P., Cornet, C., Szczap, F., Garnier, A., Pelon, J., and Meyer, K.: Impacts of cloud heterogeneities on cirrus optical properties retrieved from space-based thermal infrared radiometry, Atmos. Meas. Tech., 8, 633-647, https://doi.org/10.5194/amt-8-633-2015, 2015.

Fu, Q., Carlin, B., and Mace, G.: Cirrus horizontal inhomogeneity and OLR bias, Geophys. Res. Lett., 27, 3341-3344, 2000.

Garnier, A., Pelon, J., Dubuisson, P., Faivre, M., Chomette, O., Pascal, N., and Kratz, D. P.: Retrieval of Cloud Properties Using CALIPSO Imaging Infrared Radiometer - Part I: Effective Emissivity and Optical Depth, J. Appl. Meteorol. Clim., 51, 14071425, 2012.

Garnier, A., Pelon, J., Dubuisson, P., Yang, P., Faivre, M., Chomette, O., Pascal, N., Lucker, P., and Tim, M.: Retrieval of Cloud Properties Using CALIPSO Imaging Infrared Radiometer - Part II: effective diameter and ice water path, J. Appl. Meteorol. Clim., 52, 2582-2599, 2013.

Hogan, R. J. and Illingworth, A. J.: Parameterizing Ice Cloud Inhomogeneity and the Overlap of Inhomogeneities Using Cloud Radar Data, J. Atmos. Sci., 60, 756-767, 2003.

Hogan, R. J. and Kew, S. F.: A 3D stochastic cloud model for investigating the radiative properties of inhomogeneous cirrus clouds, Q. J. Roy. Meteor. Soc., 131, 2585-2608, 2005.

Holz, R. E., Platnick, S., Meyer, K., Vaughan, M., Heidinger, A., Yang, P., Wind, G., Dutcher, S., Ackerman, S., Amarasinghe, N., Nagle, F., and Wang, C.: Resolving ice cloud optical thickness biases between CALIOP and MODIS using infrared retrievals, Atmos. Chem. Phys., 16, 5075-5090, https://doi.org/10.5194/acp16-5075-2016, 2016.

Inoue, T.: On the temperature and effective emissivity determination of semi-transparent cirrus clouds by bi-spectral measurements in the $10 \mu \mathrm{m}$ window region, J. Meteorol. Soc. Jpn., 63, 88-99, 1985.

Kahn, B. H., Irion, F. W., Dang, V. T., Manning, E. M., Nasiri, S. L., Naud, C. M., Blaisdell, J. M., Schreier, M. M., Yue, Q., Bowman, K. W., Fetzer, E. J., Hulley, G. C., Liou, K. N., Lubin, D., Ou, S. C., Susskind, J., Takano, Y., Tian, B., and Worden, J. R.: The Atmospheric Infrared Sounder version 6 cloud products, Atmos. Chem. Phys., 14, 399-426, https://doi.org/10.5194/acp-14-3992014, 2014.
Kahn, B. H., Schreier, M. M., Yue, Q., Fetzer, E. J., Irion, F. W., Platnick, S., Wang, C., Nasiri, S. L., and L'Ecuyer, T. S.: Pixelscale assessment and uncertainty analysis of AIRS and MODIS ice cloud optical thickness and effective radius, J. Geophys. Res.Atmos., 120, 11669-11689, 2015.

Kato, S. and Marshak, A.: Solar zenith and viewing geometrydependent errors in satellite retrieved cloud optical thickness: Marine stratocumulus case, J. Geophys. Res.-Atmos., 114, D01202, https://doi.org/10.1029/2008JD010579, 2009.

Kolmogorov, A. N.: "Dissipation of Energy in the Locally Isotropic Turbulence", Proceedings of the USSR Academy of Sciences (Russian), translated into English by Kolmogorov, Andrey Nikolaevich (8 July 1991), 23, 16-18, 1941.

Kratz, D. P.: The correlated k-distribution technique as applied to the AVHRR channels, J. Quant. Spectrosc. Ra., 53, 501-517, 1995.

Lacis, A. A. and Oinas, V.: A Description of the Correlated k Distribution Method for Modeling Nongray Gaseous Absorption, Thermal Emission, and Multiple Scattering in Vertically Inhomogeneous Atmospheres, J. Geophys. Res., 96, 9027-9063, 1991.

Liou, K. N.: Influence of Cirrus Clouds on Weather and Climate Processes: A Global Perspective, Mon. Weather Rev., 114, 1167, https://doi.org/10.1175/15200493(1986)114<1167:IOCCOW>2.0.CO;2, 1986.

Lynch, D., Sassen, K., Starr, D., and Stephens, G.: Cirrus, Oxford University Press, USA, 2002.

Marchuk, G. I., Mikhailov, G. A., Nazoraliev, M. A., Dorbinjan, R. A., Kargin, B. A., and Elepov, B. S.: Monte Carlo Methods in Atmospheric Optics, in: Series in Optical Science, Vol. 12, Springer-Verlag, 1980.

Marshak, A. and Davis, A.: 3D radiative transfer in cloudy atmospheres, in: Physics of Earth and Space Environments Series, Springer-Verlag Berlin Heidelberg, 2005.

Mayer, B.: Radiative transfer in the cloudy atmosphere, Eur. Phys. J. Conferences, 1, 75-99, 2009.

McFarquhar, G. M. and Heymsfield, A. J.: Parameterization of Tropical Cirrus Ice Crystal Size Distributions and Implications for Radiative Transfer: Results from CEPEX, J. Atmos. Sci., 54, 2187-2200, 1997.

Minnis, P., Sun-Mack, S., Young, D., Heck, P., Garber, D., Chen, Y., Spangenberg, D., Arduini, R., Trepte, Q., Smith, W., Ayers, J., Gibson, S., Miller, W., Hong, G., Chakrapani, V., Takano, Y., Liou, K.-N., Xie, Y., and Yang, P.: CERES Edition-2 Cloud Property Retrievals Using TRMM VIRS and Terra and Aqua MODIS Data x2014 - Part I: Algorithms, IEEE T. Geosci. Remote, 49, 4374-4400, 2011.

Nakajima, T. and King, M. D.: Determination of the optical thickness and effective particle radius of clouds from reflected solar radiation measurements - Part I: Theory, J. Atmos. Sci., 47, 1878-1893, 1990.

Oreopoulos, L. and Cahalan, R. F.: Cloud inhomogeneity from MODIS, J. Climate, 18, 5110-5124, 2005.

Parol, F., Buriez, J. C., Brogniez, G., and Fouquart, Y.: Information Content of AVHRR Channels 4 and 5 with Respect to the Effective Radius of Cirrus Cloud Particles, J. Appl. Meteorol., 30, 973-984, 1991.

Partain, P. T., Heidinger, A. K., and Stephens, G. L.:High spectral resolution atmospheric radiative transfer: Application of the 
equivalence theorem, J. Geophys. Res.-Atmos., 105, 2163-2177, 2000.

Platnick, S., Ackerman, S. A., Baum, B. A., Heidinger, A. K., Holz, R. E., King, M. D., Menzel, W. P., Nasiri, S., Weisz, E., and Yang P.: Assessment of IDPS VIIRS Cloud Products and Recommendations for EOS-ERA CLoud CLimate Data Record Continuity, Technical report, NASA, The Suomi NPP Science Team for Clouds, 2013.

Platnick, S., King, M. D., Ackerman, S. A., Menzel, W. P., Baum, B. A., Riedi, J. C., and Frey, R. A.: The MODIS cloud products: algorithms and examples from terra, IEEE T. Geosci. Remote, 41, 459-473, 2003.

Platnick, S., Meyer, K. G., King, M. D., Wind, G., Amarasinghe, N., Marchant, B., Arnold, G. T., Zhang, Z., Hubanks, P. A., Holz, R. E., Yang, P., Ridgway, W. L., and Riedi, J.: The MODIS Cloud Optical and Microphysical Products: Collection 6 Updates and Examples From Terra and Aqua, IEEE T. Geosci. Remote, 55, 1-24, 2016.

Pujol, O.: Comment on the (misused) concept of photon in radiative transfer, and proposition of a neologism, J. Quant. Spectrosc. Ra., 159, 29-31, 2015.

Rodgers, C. D.: Inverse Methods for Atmospheric Sounding Theory and Practice, World Scientific, 2000.

Sassen, K. and Cho, B. S.: Subvisual-Thin Cirrus Lidar Dataset for Satellite Verification and Climatological Research, J. Appl. Meteorol., 31, 1275-1285, Nov. 1992.

Sassen, K., Wang, L., Starr, D., Comstock, J., and M., Q.: A midlatitude cirrus cloud climatology form the facility for atmospheric remote sensing - Part V: Cloud Structural Properties?, J. Atmos. Sci., 64, 2483-2501, 2007.

Sassen, K., Wang, Z., and Liu, D.: Global distribution of cirrus clouds from CloudSat/Cloud-Aerosol Lidar and Infrared Pathfinder Satellite Observations (CALIPSO) measurements, J. Geophys. Res.-Atmos., 113, D00A12, https://doi.org/10.1029/2008JD009972, 2008.

Smith, S. A. and Del Genio, A. D.: Analysis of Aircraft, Radiosonde, and Radar Observations in Cirrus Clouds Observed during FIRE II: The Interactions between Environmental Structure, Turbulence, and Cloud Microphysical Properties, J. Atmos. Sci., 58, 451-461, 2001.

Stephens, G. L., Gabriel, P. M., and Tsay, S.: Statistical radiative transport in one-dimensional media and its application to the terrestrial atmosphere, Transport Theor. Stat., 20, 139-175, 1991.

Szczap, F., Isaka, H., Saute, M., Guillemet, B., and Gour, Y.: Inhomogeneity effects of 1D and 2D bounded cascade model clouds on their effective radiative properties, Phys. Chem. Earth Pt. B, 25, 83-89, 2000.

Szczap, F., Gour, Y., Fauchez, T., Cornet, C., Faure, T., Jourdan, O., Penide, G., and Dubuisson, P.: A flexible three-dimensional stratocumulus, cumulus and cirrus cloud generator (3DCLOUD) based on drastically simplified atmospheric equations and the Fourier transform framework, Geosci. Model Dev., 7, 17791801, https://doi.org/10.5194/gmd-7-1779-2014, 2014.

Varnai, T. and Marshak, A.: Statistical analysis of the uncertainties in cloud optical depth retrievals caused by three-dimensional radiative effects, J. Atmos. Sci., 58, 1540-1548, 2001.
Wang, C., Yang, P., Baum, B., Platnick, S., Heidinger, A. K., Hu, Y., and Holz, R. E.: Retrieval of ice cloud optical thickness and effective size using a fast infrared radiative transfer model, J. Appl. Meteorol. Clim., 50, 2283-2297, 2011.

Wang, C., Platnick, S., Zhang, Z., Meyer, K., Wind, G., and Yang, P.: Retrieval of ice cloud properties using an optimal estimation algorithm and MODIS infrared observations - Part II: Retrieval evaluation, J. Geophys. Res.-Atmos., 121, 5827-5845, https://doi.org/10.1002/2015JD024528, 2016a.

Wang, C., Platnick, S., Zhang, Z., Meyer, K., and Yang, P.: Retrieval of ice cloud properties using an optimal estimation algorithm and MODIS infrared observations - Part I: Forward model, error analysis, and information content, J. Geophys. Res.-Atmos., 121, 5809-5826, https://doi.org/10.1002/2015JD024526, $2016 \mathrm{~b}$.

Wang, L. and Sassen, K.: Wavelet Analysis of Cirrus Multiscale Structures from Lidar Backscattering: A Cirrus Uncinus Complex Case Study, J. Appl. Meteorol. Climatol., 47, 2645-2658, 2008.

Winker, D. M., Vaughan, M. A., Omar, A., Hu, Y., Powell, K. A., Liu, Z., Hunt, W. H., and Young, S. A.: Overview of the CALIPSO Mission and CALIOP Data Processing Algorithms, J. Atmos. Ocean. Tech., 26, 2310-2323, https://doi.org/10.1175/2009JTECHA1281.1, 2009.

Wood, R. and Taylor, J. P.: Liquid water path variability in unbroken marine stratocumulus cloud, Q. J. Roy. Meteor. Soc., 127, 26352662, 2001.

Yang, P., Zhang, L., Hong, G., Nasiri, S., Baum, B., Huang, H., King, M., and Platnick, S.: Differences between collection 4 and 5 MODIS ice cloud optical/microphysical products and their impact on radiative forcing simulations, IEEE T. Geosci. Remote., 45, 2886-2899, 2007.

Yang, P., Bi, L., Baum, B., Liou, K.-N., Kattawar, G., Mishchenko, M., and Cole, B.: Spectrally consistent scattering, absorption, and polarization properties of atmospheric ice crystals at wavelengths from 0.2 to $100 \mu \mathrm{m}$, J. Atmos. Sci., 70, 330-347, 2013.

Zhang, Z. and Platnick, S.: An assessment of differences between cloud effective particle radius retrievals for marine water clouds from three MODIS spectral bands, J. Geophys. Res.-Atmos., 116, D20215, https://doi.org/10.1029/2011JD016216, 2011.

Zhang, Z., Ackerman, A. S., Feingold, G., Platnick, S., Pincus, R., and Xue, H.: Effects of cloud horizontal inhomogeneity and drizzle on remote sensing of cloud droplet effective radius: Case studies based on large-eddy simulations, J. Geophys. Res.Atmos., 117, D19208, https://doi.org/10.1029/2012JD017655, 2012.

Zinner, T. and Mayer, B.: Remote sensing of stratocumulus clouds: Uncertainties and biases due to inhomogeneity, J. Geophys. Res., 111, D14209, https://doi.org/10.1029/2005JD006955, 2006.

Zinner, T., Wind, G., Platnick, S., and Ackerman, A. S.: Testing remote sensing on artificial observations: impact of drizzle and 3-D cloud structure on effective radius retrievals, Atmos. Chem. Phys., 10, 9535-9549, https://doi.org/10.5194/acp10-9535-2010, 2010. 